\title{
TEVAR for Symptomatic Stanford B Dissection: A Systematic Review of 30-Day Mortality and Morbidity
}

\author{
Michael Ramdass ${ }^{1}$ \\ ${ }^{1}$ Department of Clinical Surgical Sciences, University of the West \\ Indies, Port of Spain General Hospital, Port of Spain, Trinidad and \\ Tobago \\ Thorac Cardiovasc Surg 2015;63:97-112.
}

\begin{abstract}
Address for correspondence Michael Ramdass, FRCS, FACS, Department of Clinical Surgical Sciences, University of the West Indies, Port of Spain General Hospital, Charlotte Street, Port of Spain West Indies, Trinidad and Tobago (e-mail: jimmyramdass@gmail.com).
\end{abstract}

\begin{abstract}
Keywords

- endovascular repair

- Stanford type B dissection

- acute and chronic aortic dissection

Background The aim of this study was to determine morbidity and 30-day mortality rates of thoracic endovascular aortic repair (TEVAR) for Stanford B dissection over a 16year period and determine if these rates have improved with better stent-graft technology and surgical technique.

Methods Electronic databases were searched in all languages and a systematic review conducted. A comparison of the early (1998-2007 = 787 patients) and later (2007$2013=787$ patients) halves of the patient population was done. Studies were chosen based on availability of details regarding morbidity and mortality. Ambiguous studies were excluded.

Results A total of 69 suitable studies published between 1998 and 2013 (1,574 patients) were examined including 1 randomized control trial, 55 retrospective studies, 3 prospective, 1 mixed, and 9 case reports. Overall mortality and morbidity rates for TEVAR was $8.07 \%(n=127)$ and $30.8 \%(n=485)$, respectively. The stent-graft-related death rate was $6.20 \%$ (97 cases excluding medically related deaths). The endoleak rate was $5.9 \%$ of which most were type I. Major complications include stroke $(2.7 \%)$, paraplegia (1.9\%), partial thrombosis of false lumen (2.5\%), retrograde type A dissection (3.1\%), visceral malperfusion (2.0\%), conversion to open intervention $(1.9 \%)$, and secondary intervention (4.1\%). The stent-graft-related mortality rate increased in the 2007 to 2013 group compared with the 1998 to 2007 group (56.2 vs. 24\% of patients who died; $p<0.05$ ). There was also an increase in the overall morbidity rates from $25 \%$ (1998-2007) to 36.6\% (2007-2013) but did not reach statistical significance $p>0.05$. Conclusion Mortality and morbidity rates for TEVAR seemed to have increased over the past 16 years despite improved technology and surgical technique. This may be explained by the increasing liberal use of TEVAR intervention and quite possibly better reporting. The current data are highly heterogenous making it difficult for solid conclusions to be drawn. The only way forward is through better data registries and well-designed clinical trials.
\end{abstract}

received

August 11, 2013

accepted after revision

December 18, 2013

published online

April 21, 2014 (c) 2015 Georg Thieme Verlag KG

Stuttgart · New York
DOI http://dx.doi.org/ 10.1055/s-0034-1370760. ISSN 0171-6425. 


\section{Introduction}

The first endoluminal procedure was conducted by Dotter and Judkins in $1964^{1}$ and progressed slowly up to 1991 when Parodi et $\mathrm{al}^{2}$ made significant advancements in the treatment of abdominal aortic aneurysms. Techniques and technology evolved over the years and spread into the various branches of vascular surgery. Up to 2013, major studies on type B dissection included meta-analyses by Eggebrecht et $\mathrm{al}^{3}$ in 2006, Walsh et $\mathrm{al}^{4}$ in 2008, Xiong et al in 2009, ${ }^{5}$ and Zhang et al in $2012,{ }^{6}$ as well as the INSTEAD ${ }^{7,8}$ and IRAD $^{9}$ trials by Nienaber et al and Fattori et al, respectively. This article is intended to be a systematic review of available data for 16 years from 1998 to 2013 with regard to morbidity and mortality with a comparison of the first and later halves of the patient population to determine if rates have improved with the advent of improved technology and surgical techniques.

\section{Methodology}

A systematic review was conducted using separate search strategies in the PubMed, MeSH thesaurus, and Cochrane databases. The following registries were included: EUROSTAR Registry, United Kingdom Thoracic Endograft Registry, and International Registry of Acute Aortic Dissection. The PRISMA guidelines were followed as recommended for systematic review reporting.

\section{PICOS Statement}

The study was conducted focusing on the 30-day mortality and morbidity of symptomatic type B acute and/or chronic aortic dissections (S-B-AD). Suitable studies for 16 years from 1998 to 2013 were selected for analysis. The objectives of the study were to determine overall rates for the 16 -year period and to perform a comparison of the first (787 patients from 1998 to 2007) and later (787 patients from 2007 to 2013) halves of the patient population to determine if rates have improved with the advent of improved technology and surgical techniques.

\section{Protocol and Registration}

No review protocol was created online.

\section{Eligibility Criteria}

Suitable studies were chosen based on the accuracy of documentation of 30-day mortality and morbidity specifically for the S-B-AD. Once the information was clearly documented, the study was included.

\section{Information Sources}

A systematic review was conducted using separate search strategies in the following databases: PubMed, MeSH thesaurus, and Cochrane databases. The Embase, Ingenta, Zetoc, and Ovid databases were also used as well as the following registries were included: EUROSTAR Registry, United Kingdom Thoracic Endograft Registry, and International Registry of Acute Aortic Dissection.

\section{Search}

A standard PubMed search using the "Advanced Search Option" for matching terms was first used to obtain the meta-analyses, review articles, and case series on the treatment of S-B-AD, whether acute or chronic as well as involving consequent aneurysm formation or not.

The search topics included the following:

1. "Type B dissection and descending thoracic dissection"

2. "Medical treatment"

3. "Open surgery"

4. "Endovascular/stent repair"

Combinations were done using the "AND" option. This obtained the following results:

- "Type B dissection and descending thoracic dissection" yielded 340 articles.

- The 340 articles were then combined using the "AND" option in the search box yielding 108 for medical treatment, 69 for open surgery, and 44 for endovascular/stent repair. There were 51 suitable articles selected for analysis.

Another search strategy was employed by searching through a MeSH search in PubMed combining "Aortic Aneurysm, Thoracic [MeSH]" AND "Stents [MeSH]" AND "Aneurysm, dissecting [MeSH]." This yielded 441 articles from which the search was confined to clinical trials (three selected), review articles (two selected), randomized controlled trials (one selected), and meta-analyses (six selected).

The databases from the Cochrane library, Embase, Ingenta, Zetoc, and Ovid did not yield any new relevant information; however, hand-searching relevant references obtained via a standard PubMed search for the topic yielded a further 14 relevant articles.

Cumulatively, 69 suitable studies were selected. The six meta-analyses and two reviews were excluded to avoid duplicating data ( - Fig. 1). A total of 1,574 patients were treated for S-B-AD. There were no ethical considerations. Results were entered into SPSS 20 (IBM SPSS 20 STATISTIC, United States) and analyzed using Pearson chi-square testing.

\section{Results}

A total of 69 articles with 1,574 patients were selected for the 16-year period, 1998 to 2013. Most included a mix of various types of descending thoracic aortic pathology including acute rupture, chronic aneurysmal disease, Stanford type A and B dissections, and traumatic disruption. Selected articles contained a detailed breakdown of the results, patient demographics, and details of complications associated with S-B-AD (-Table 1).

Thoracic endovascular aortic repairs (TEVARs) were done in the following countries: 11 (16\%) studies from China, Japan, and Korea in the Far East; 16 (23\%) from the United States; and 38 (55\%) from European region including Germany, Austria, France, United Kingdom, Switzerland, Norway, Sweden, Italy, Belgium, and Denmark and Turkey. There were two from Brazil and one from New Zealand and Canada each (6\%). 


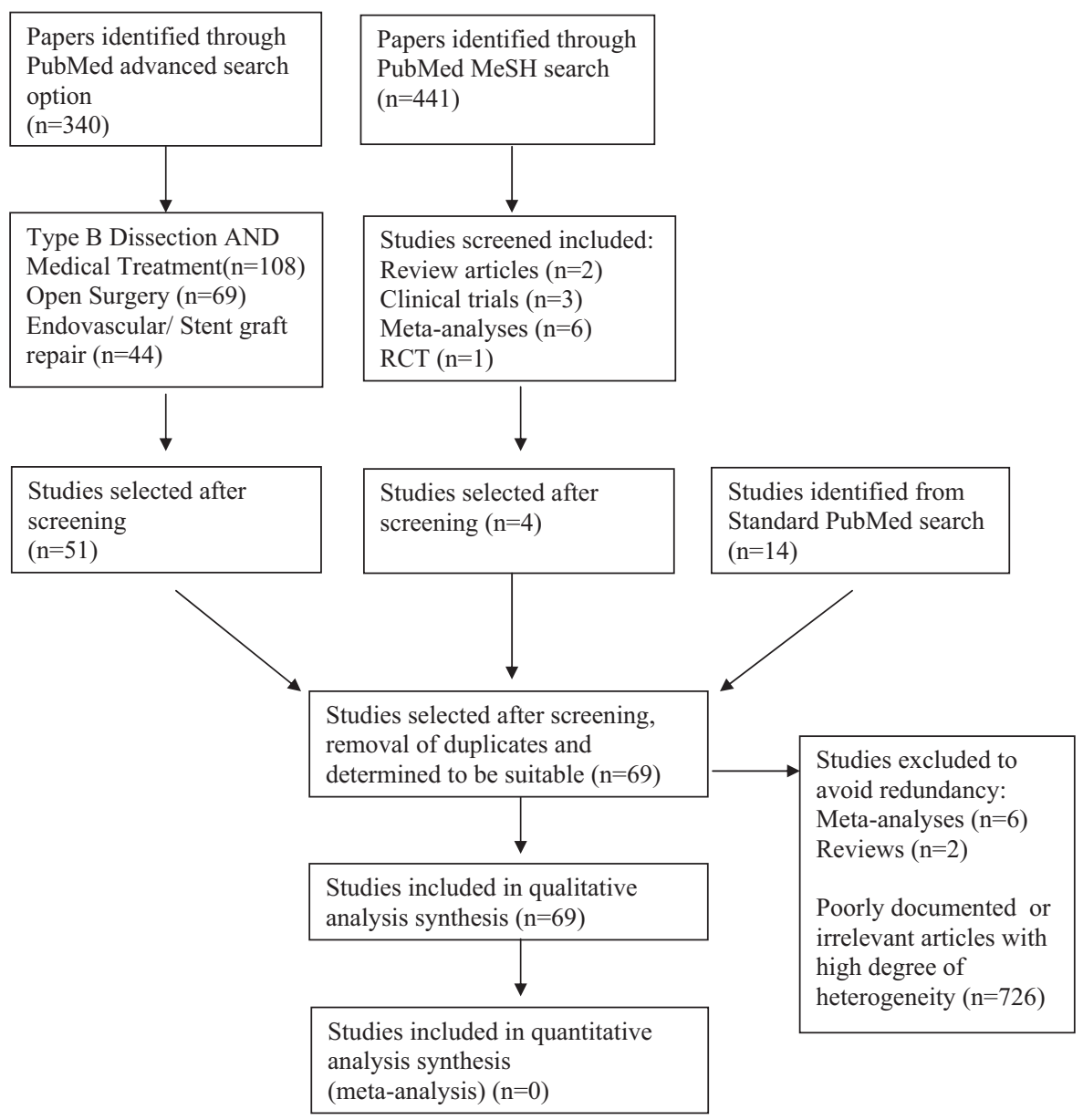

Fig. 1 PRISMA flow diagram showing search strategy.

The largest series were from Kische et al, ${ }^{10}$ Leurs et al, ${ }^{11}$ and Nienaber et $\mathrm{al}^{7}$ with 171,106 , and 72 cases, respectively. The other studies contained small and intermediate sized populations and nine case reports were also included. Tests for heterogeneity in patient demographics in terms of age, ASA (American Society of Anaesthesiologists Physical Status Classification) grade, and comorbidities were not conducted because there was great variation in the way in which data were recorded.

The mean age range was 62 years with a range of 54 to 75 , a median of 60 , and mode of 59 with the age stated in only 36 articles (52\%). Studies selected included the following according to year: $1998=1,1999=2,2000=4,2001=3$, $2002=7, \quad 2003=7, \quad 2004=7, \quad 2005=2, \quad 2006=7$, $2007=6, \quad 2008=10, \quad 2009=6, \quad 2010=0, \quad 2011=2$, $2012=2$, and $2013=3$.

In terms of device, the most commonly used devices were the Medtronic Talent (Medtronic, Minneapolis, Minnesota, United States) and Gore TAG Thoracic Endoprosthesis (Gore Medical, Flagstaff, Arizona, United States) devices in 30 studies (43\%) followed by the Zenith Excluder (Cook Medical, Bloomington, Indiana, United States) and the Valiant (Medtronic, Minneapolis, Minnesota, United States) types. The device used was not stated in 36 studies.

Of 1,574 patients treated for Stanford B dissections, the overall 30-day mortality was $8.07 \%(n=127)(-$ Table $\mathbf{1})$.
These were related to a variety of causes including procedurerelated false aneurysm rupture, retrograde type A dissection, ruptured type I endoleak, persistent visceral and lower limb malperfusion, aorto-esophageal fistula, further type B dissection, conversion to open surgery, as well as ruptured access vessels. There were $24(1.52 \%)$ medical-related death within 30 days. The total number of deaths directly related to stenting was 97 (6.20\%) (excluding medical-related deaths) within 30 days of TEVAR (- Table $\mathbf{2}$ ).

Procedure-related morbidities included retrograde type A dissection (49), visceral malperfusion (32), lower limb ischemia (5), aorto-esophageal fistula (4), stroke (41), paraplegia/ paraparesis (30), type I endoleak (82), type II endoleak (11), partial thrombosis of the false lumen (39), conversion to open surgery failed procedure (30), secondary interventions (65), renal failure (31), device migration/modular disconnection/ junctional endoleaks (7), emboli (4), overstenting of the celiac artery (1), postimplantation syndrome (3), and failure (2). The overall morbidity rate for the patient population was $30.8 \%$ (-Table 3).

With regard to the comparison of the 1998 to 2007 period (787 patients) with the 2007 to 2013 period (787 patients), the findings were quite significant. The stent-graft-related mortality rates (which includes the subset of patients who died; $n=127$ ) doubled in the 2007 to 2013 period versus the 1998 to 2007 period; 56.2 versus $24.0 \%$, respectively 


\begin{tabular}{|c|c|c|c|c|c|}
\hline & 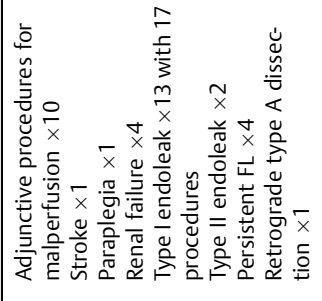 & 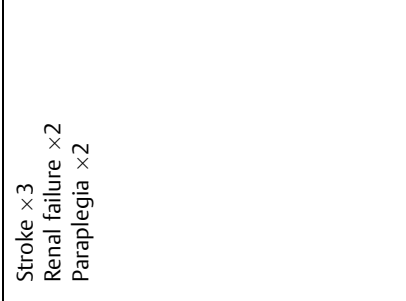 & 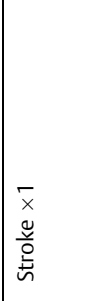 & 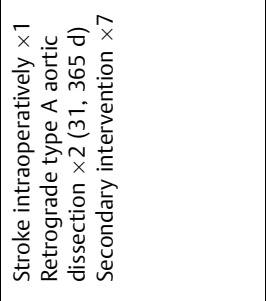 & 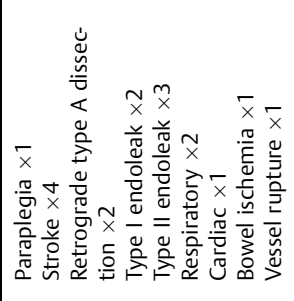 \\
\hline 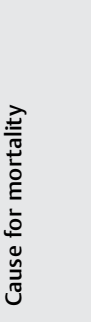 & 1 & 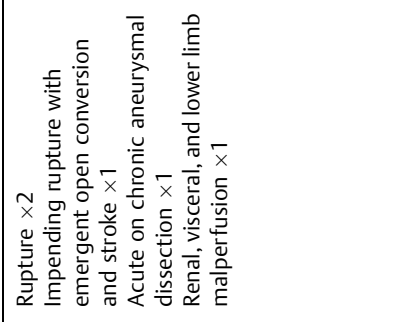 & & 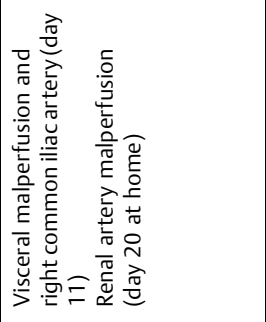 & 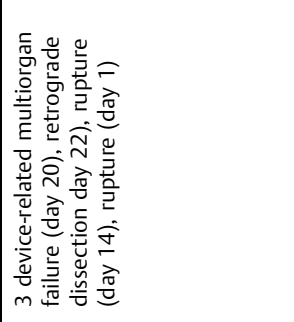 \\
\hline 苞 & 0 & in & 0 & $\sim$ & $\checkmark$ \\
\hline$z$ & in & g & $a$ & $\stackrel{\circ}{m}$ & 9 \\
\hline 芩 & 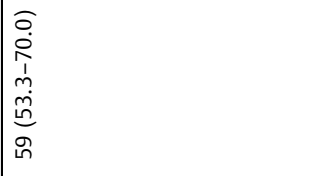 & \begin{tabular}{l}
$\stackrel{m}{\stackrel{m}{2}}$ \\
$+H$ \\
\hdashline$\dot{i}$
\end{tabular} & $\begin{array}{l}\text { in } \\
+1 \\
i\end{array}$ & 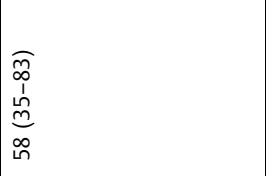 & 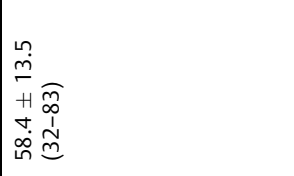 \\
\hline هัّ & 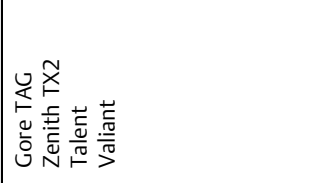 & 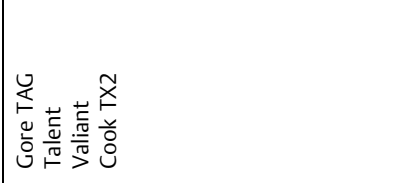 & $\Sigma$ & $\begin{array}{l}x \\
\qquad \\
\text { 壳 } \\
\text { N } \\
\text { N }\end{array}$ & 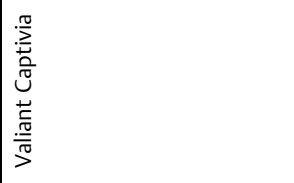 \\
\hline : & 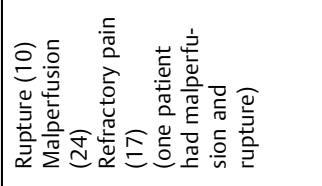 & 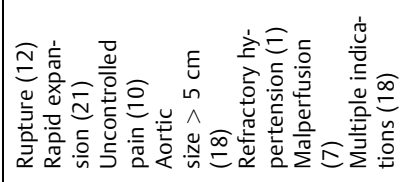 & 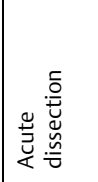 & 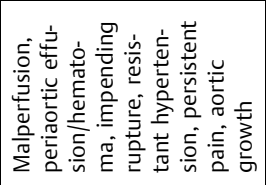 & 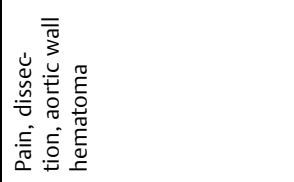 \\
\hline 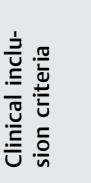 & 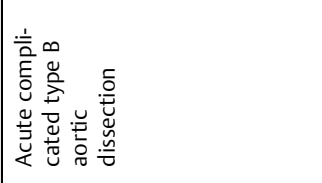 & 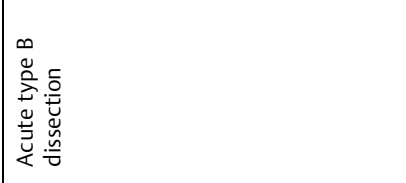 & 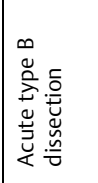 & 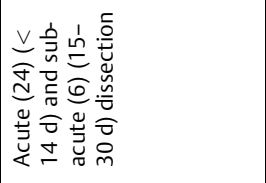 & 焉 \\
\hline 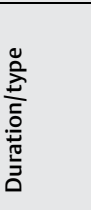 & 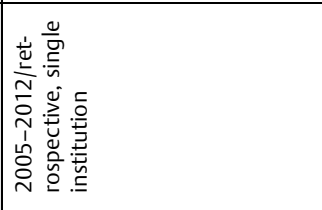 & 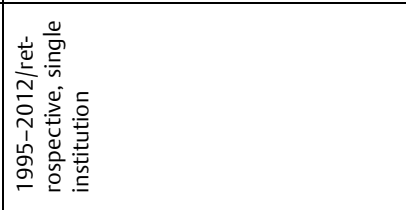 & 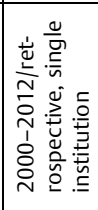 & 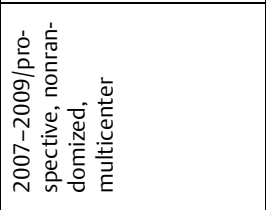 & 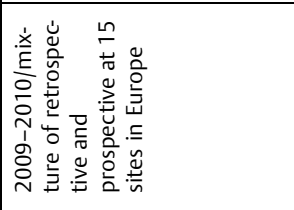 \\
\hline 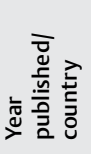 & 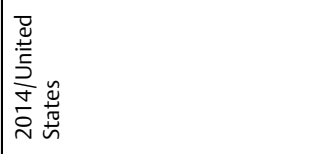 & 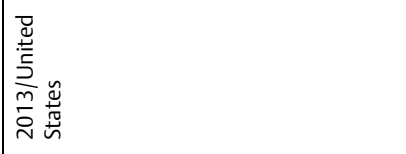 & 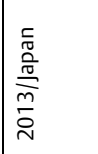 & 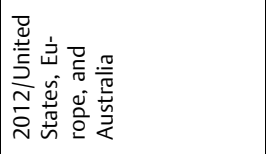 & 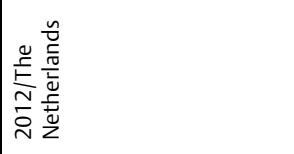 \\
\hline 旁 & 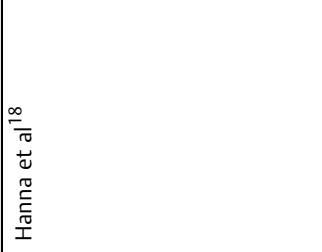 & 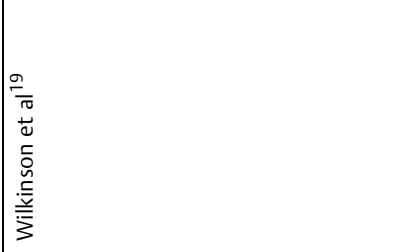 & 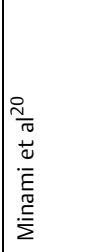 & 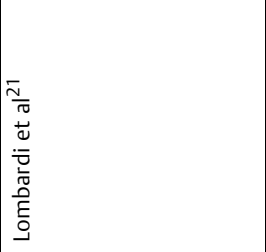 & 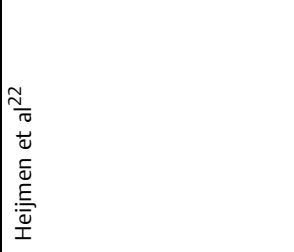 \\
\hline
\end{tabular}




\begin{tabular}{|c|c|c|c|c|c|}
\hline 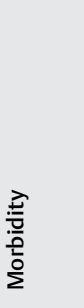 & 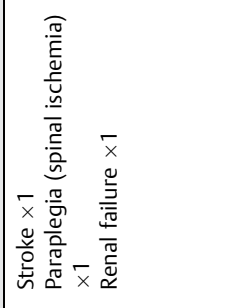 & 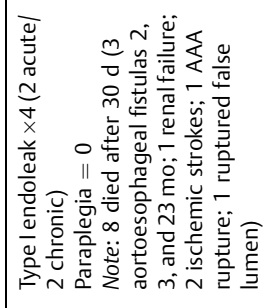 & 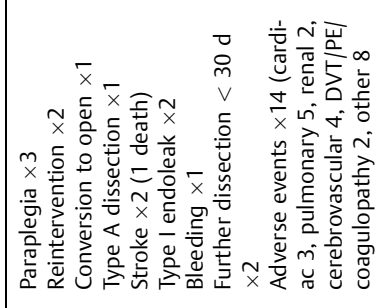 & 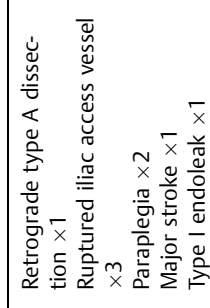 & 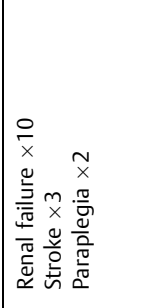 \\
\hline 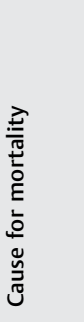 & 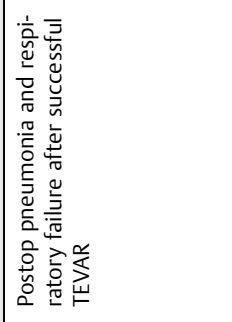 & 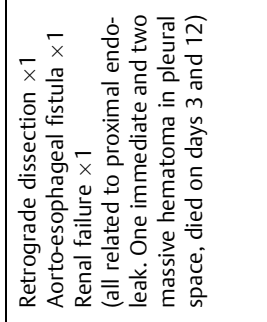 & 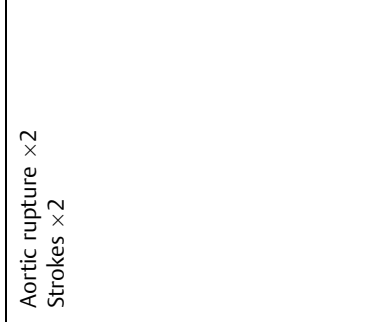 & 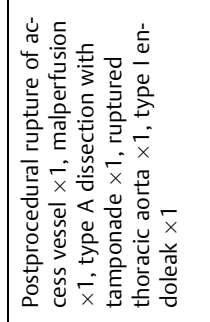 & 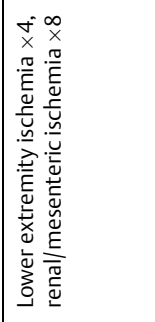 \\
\hline 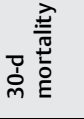 & - & $m$ & $m$ & in & $\simeq$ \\
\hline$z$ & o & 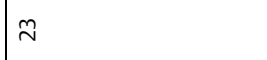 & 9 & $\approx$ & 8 \\
\hline 胥 & 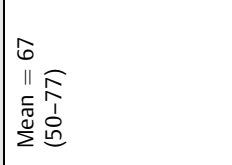 & 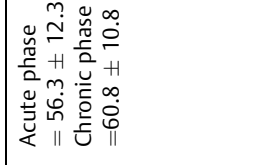 & 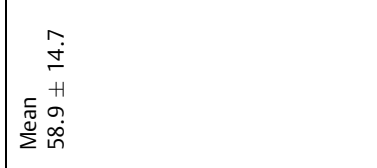 & $\begin{array}{l}\widehat{n} \\
\hat{1} \\
\underline{\underline{0}} \\
\infty \\
\dot{0} \\
\bar{b}\end{array}$ & $\begin{array}{l}\infty \\
\dot{b} \\
\dot{\omega}\end{array}$ \\
\hline$\stackrel{\check{\Sigma}}{\bar{\Delta}}$ & 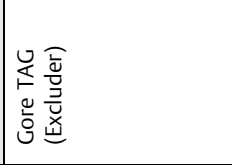 & 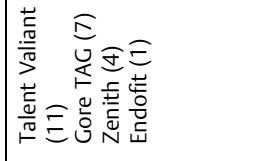 & 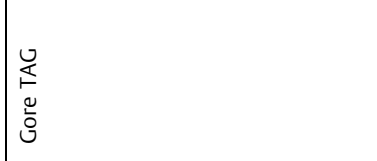 & $\mid \begin{array}{l}\frac{\vec{c}}{\tilde{\sigma}} \\
\frac{\vec{v}}{\sigma}\end{array}$ & $\tilde{z}$ \\
\hline 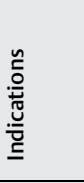 & 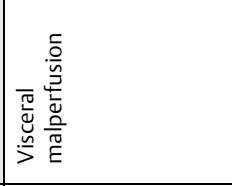 & 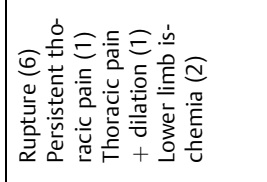 & 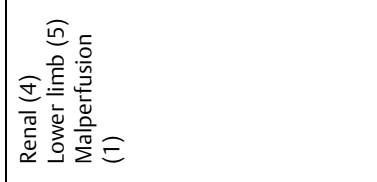 & 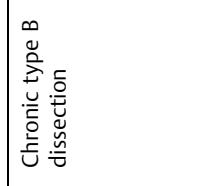 & 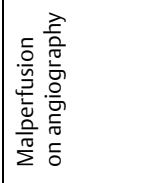 \\
\hline 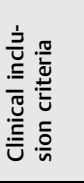 & 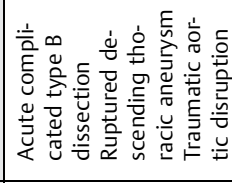 & 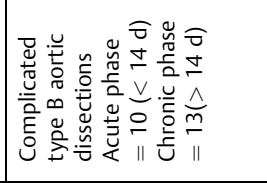 & 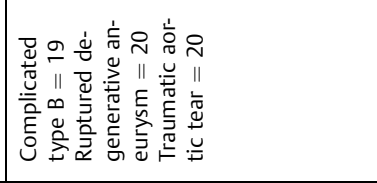 & 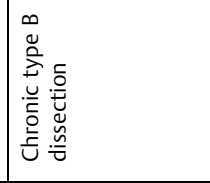 & 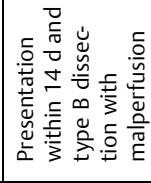 \\
\hline 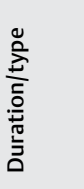 & 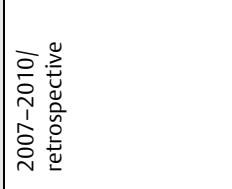 & 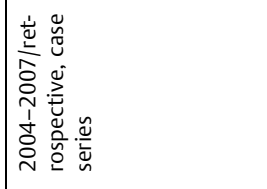 & 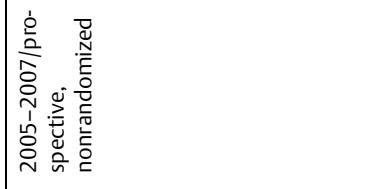 & 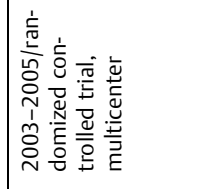 & 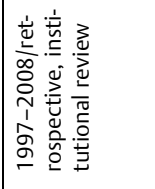 \\
\hline 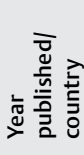 & 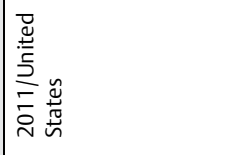 & 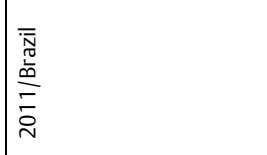 & 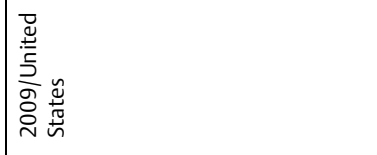 & | & 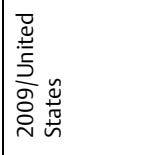 \\
\hline $\begin{array}{l}\text { 高 } \\
\text { 旁 }\end{array}$ & 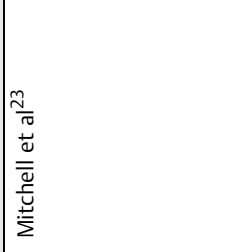 & 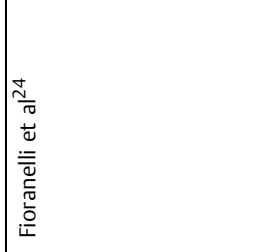 & 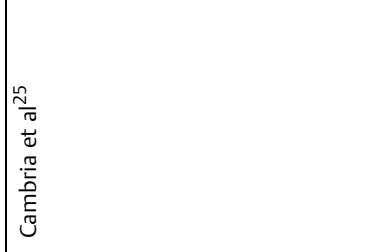 & 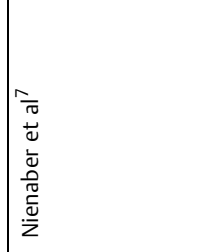 & 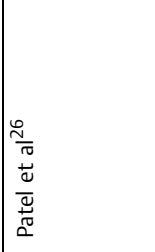 \\
\hline
\end{tabular}




\begin{tabular}{|c|c|c|c|c|c|c|c|}
\hline 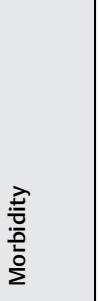 & 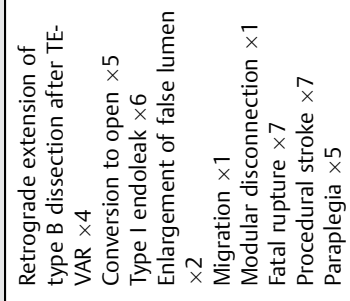 & $\tilde{z}$ & 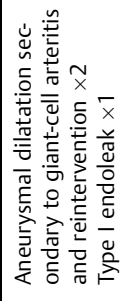 & 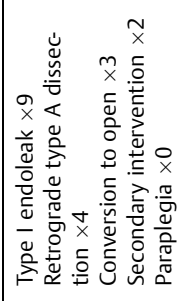 & 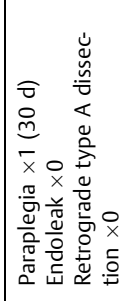 & 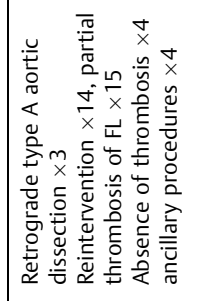 & 0 \\
\hline 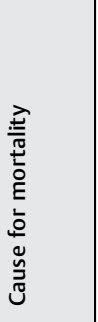 & 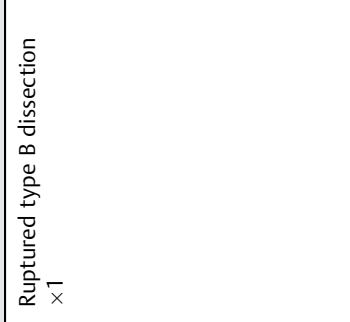 & 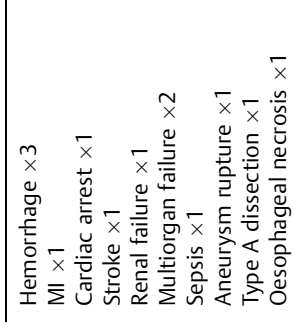 & 1 & 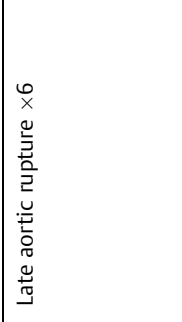 & 1 & 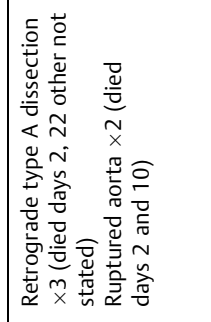 & \\
\hline 竞 & $\sigma$ & r & 0 & 0 & 0 & in & 0 \\
\hline$z$ & $\Sigma$ & $\stackrel{g}{m}$ & - & $\stackrel{\infty}{m}$ & $=$ & $\stackrel{n}{m}$ & - \\
\hline 娄 & 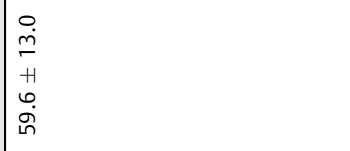 & $\Sigma$ & $\stackrel{m}{n}$ & $\tilde{z}$ & 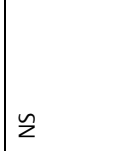 & $\tilde{z}$ & $\tilde{z}$ \\
\hline 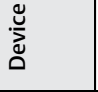 & \begin{tabular}{|l}
$\overrightarrow{\vec{v}}$ \\
$\frac{\vec{\omega}}{r}$
\end{tabular} & $\tilde{z}$ & $\mid \begin{array}{l}\overrightarrow{\underline{w}} \\
\frac{\vec{w}}{\sqrt{\sigma}}\end{array}$ & $\tilde{z}$ & 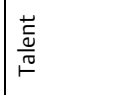 & $\Sigma$ & $\tilde{z}$ \\
\hline 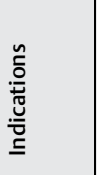 & 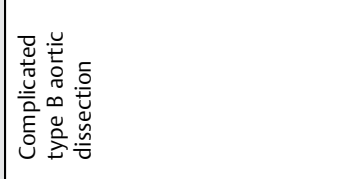 & 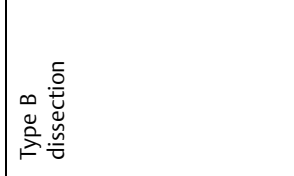 & 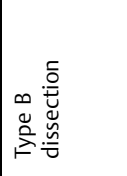 & 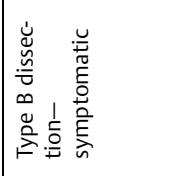 & 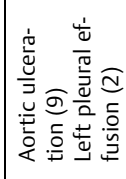 & 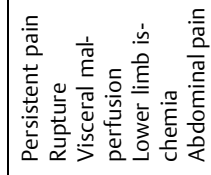 & 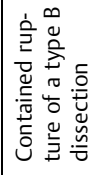 \\
\hline 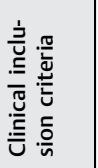 & 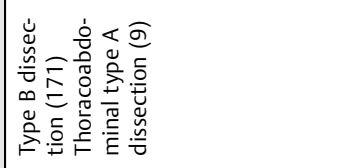 & 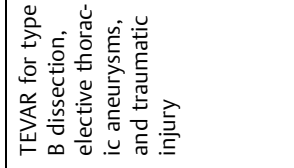 & 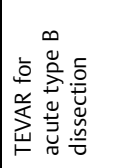 & 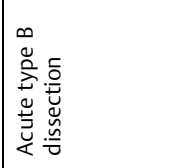 & 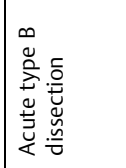 & 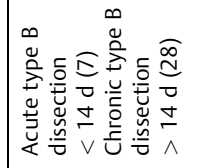 & 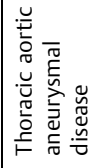 \\
\hline 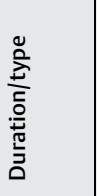 & 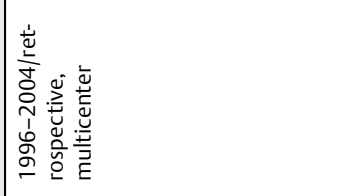 & 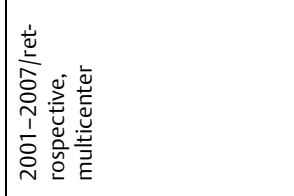 & 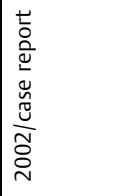 & 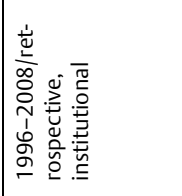 & 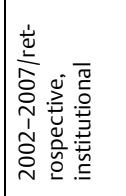 & 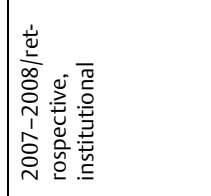 & 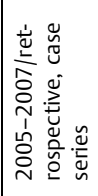 \\
\hline 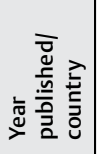 & 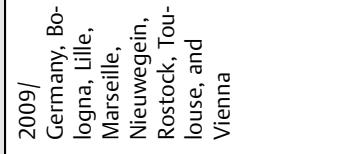 & 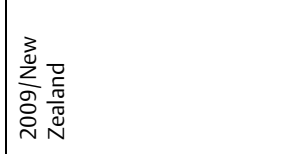 & 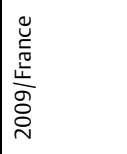 & 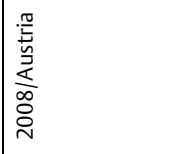 & 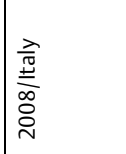 & 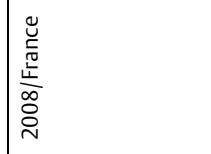 & 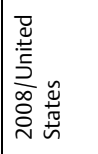 \\
\hline $\begin{array}{l}\text { 毫 } \\
\frac{3}{4}\end{array}$ & 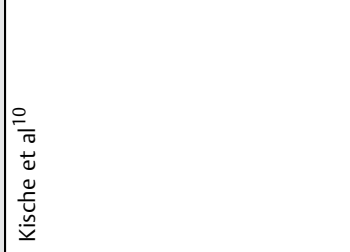 & 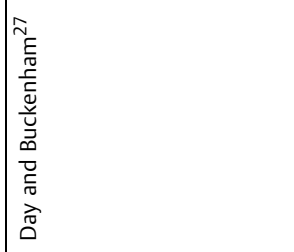 & 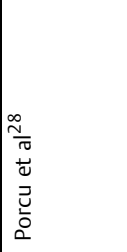 & 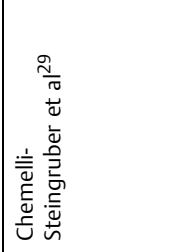 & 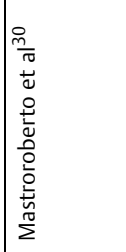 & 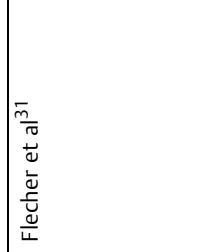 & $\begin{array}{l}\frac{\tilde{y}}{\pi} \\
\frac{0}{0} \\
\frac{\omega}{2} \\
\frac{0}{2}\end{array}$ \\
\hline
\end{tabular}




\begin{tabular}{|c|c|c|c|c|c|c|c|c|c|c|c|}
\hline 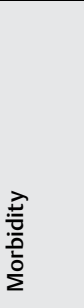 & 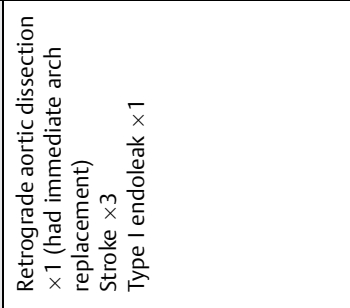 & 0 & 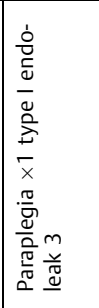 & 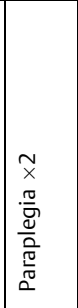 & 0 & 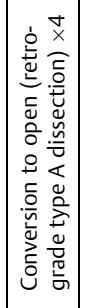 & 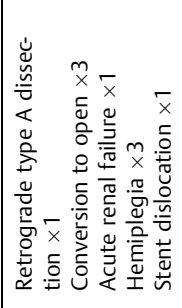 & 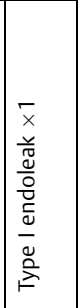 & 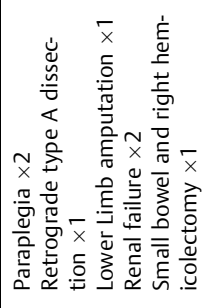 & 0 & 0 \\
\hline 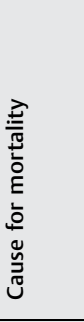 & 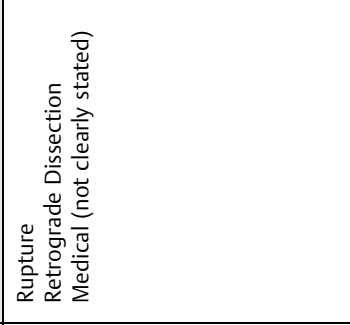 & 1 & 1 & $\bar{\Sigma}$ & 1 & 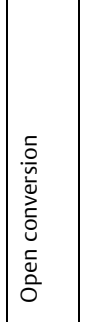 & 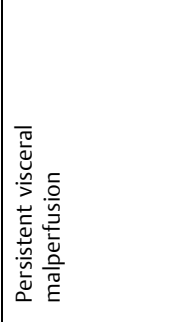 & 1 & 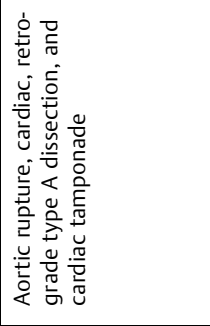 & & 1 \\
\hline 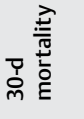 & r & 0 & - & $m$ & 0 & - & $\sim$ & 0 & $m$ & 0 & 0 \\
\hline$z$ & 6 & $\nabla$ & $\sigma$ & $\sqrt{\lambda}$ & - & $\stackrel{\infty}{\sim}$ & $\stackrel{m}{q}$ & 0 & I & - & m \\
\hline 茕 & $\tilde{z}$ & 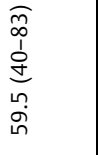 & 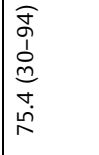 & 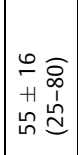 & i̊n & $\tilde{z}$ & 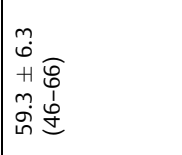 & $\begin{array}{l}\frac{1}{n} \\
i \\
0 \\
0 \\
6 \\
6\end{array}$ & $\begin{array}{c}\tilde{m} \\
0 \\
1 \\
\tilde{m} \\
\tilde{m} \\
i n\end{array}$ & in & $\tilde{z}$ \\
\hline ֻัّ & 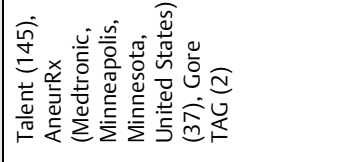 & 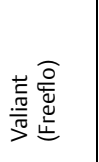 & $\tilde{z}$ & $\tilde{z}$ & 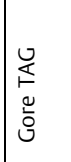 & $\tilde{z}$ & 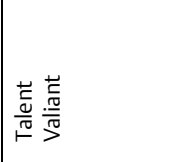 & $\tilde{z}$ & 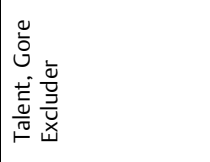 & 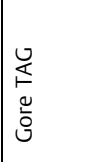 & 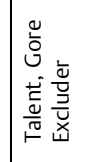 \\
\hline & 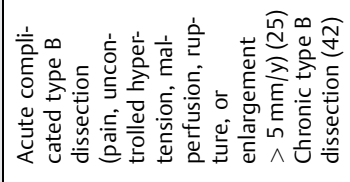 & 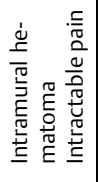 & 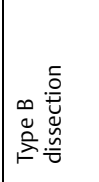 & 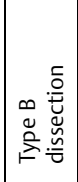 & 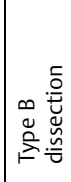 & 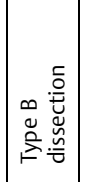 & 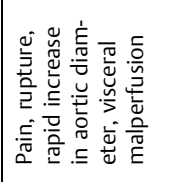 & 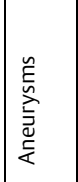 & 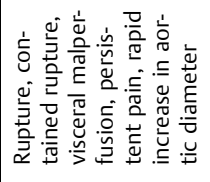 & 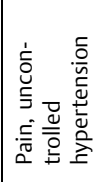 & 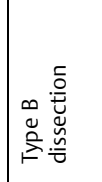 \\
\hline 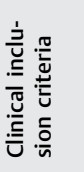 & 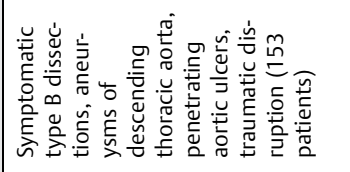 & 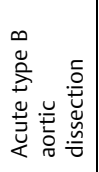 & 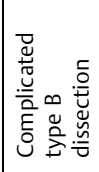 & 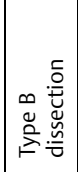 & 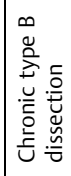 & 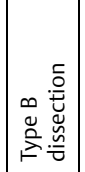 & 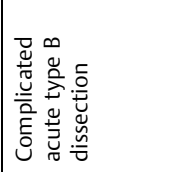 & 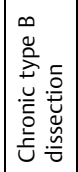 & 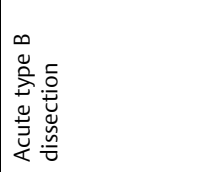 & 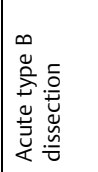 & 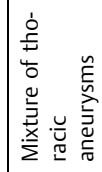 \\
\hline 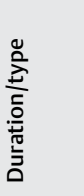 & 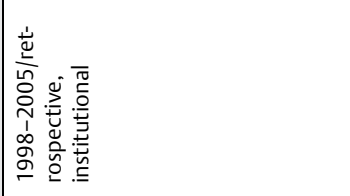 & 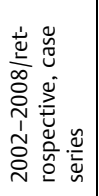 & 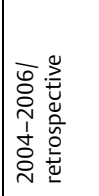 & 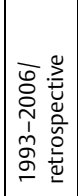 & 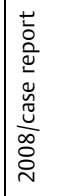 & 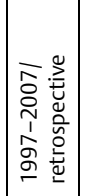 & 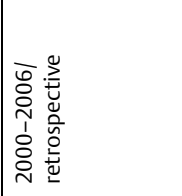 & 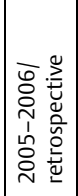 & 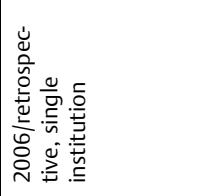 & 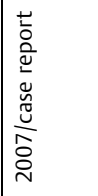 & 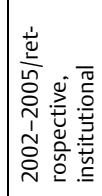 \\
\hline 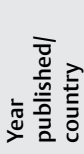 & 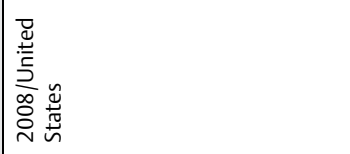 & 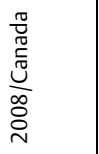 & 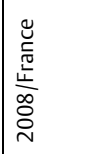 & 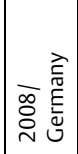 & 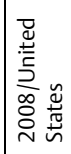 & 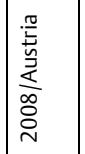 & 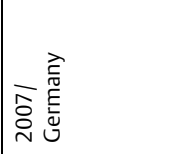 & 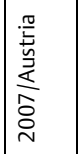 & 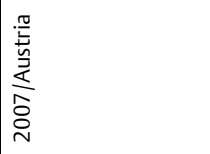 & 兰 & 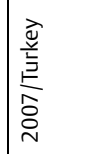 \\
\hline $\begin{array}{l}\text { कo } \\
\text { 章 }\end{array}$ & 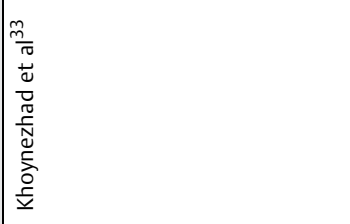 & 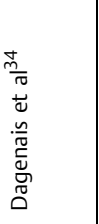 & 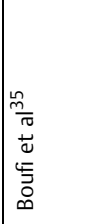 & 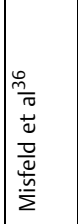 & 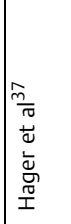 & 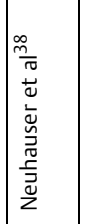 & 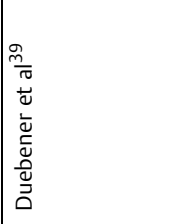 & 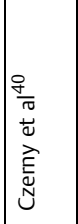 & 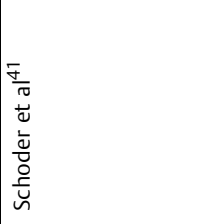 & 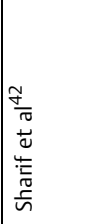 & 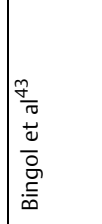 \\
\hline
\end{tabular}




\begin{tabular}{|c|c|c|c|c|c|c|c|c|}
\hline 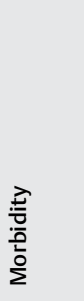 & 0 & 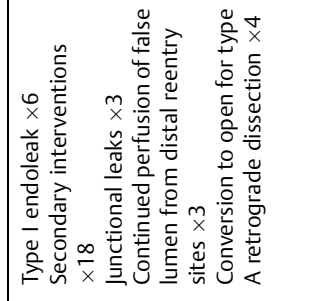 & 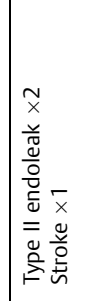 & 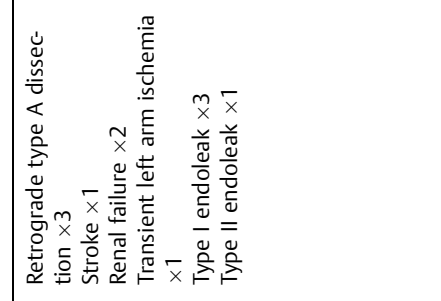 & 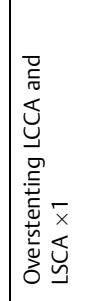 & 0 & 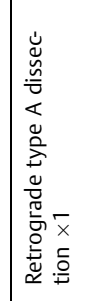 & 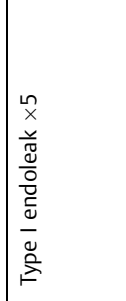 \\
\hline 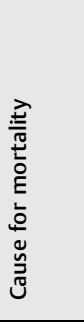 & 1 & 1 & 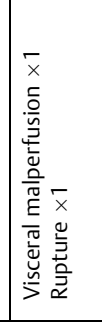 & 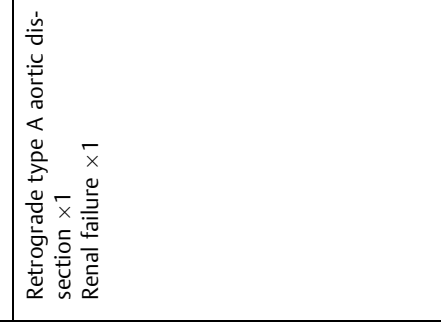 & 1 & 1 & 1 & 1 \\
\hline 竞 & 0 & 0 & $\sim$ & $\sim$ & 0 & 0 & 0 & $m$ \\
\hline$z$ & - & F & $\simeq$ & 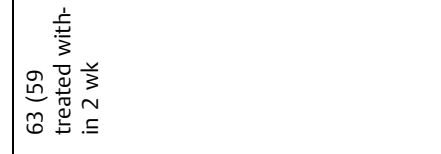 & - & $m$ & in & $\stackrel{\infty}{m}$ \\
\hline 娄 & 6 & 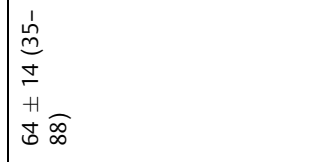 & $\tilde{z}$ & $\tilde{z}$ & (ด) & $\tilde{z}$ & $\tilde{z}$ & $\tilde{z}$ \\
\hline هั & 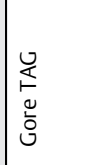 & 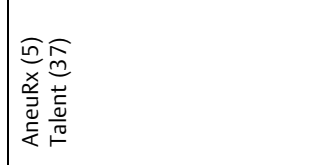 & 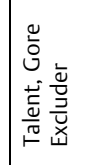 & 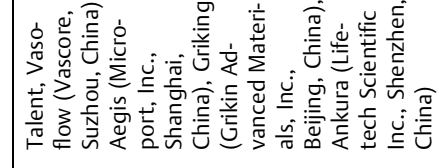 & $\Sigma$ & $\Sigma$ & 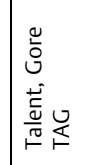 & 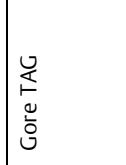 \\
\hline 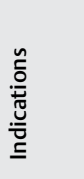 & 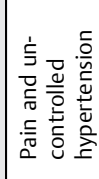 & 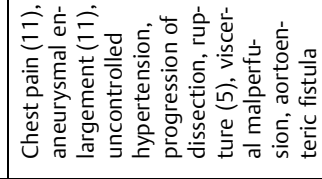 & 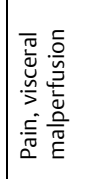 & 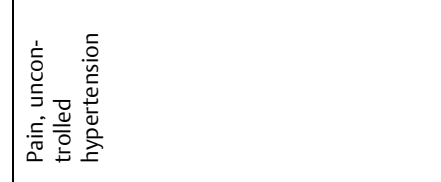 & 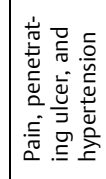 & 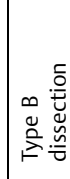 & 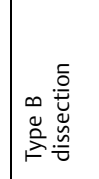 & 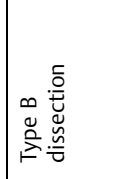 \\
\hline 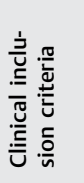 & 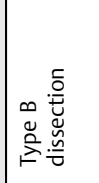 & 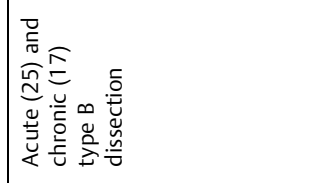 & 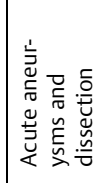 & 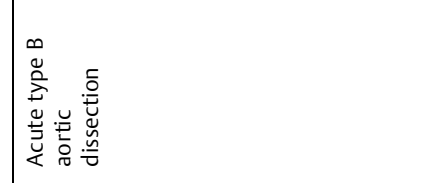 & 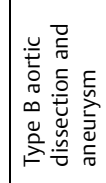 & 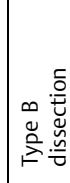 & 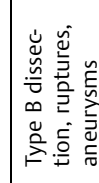 & 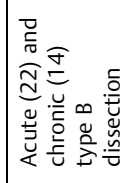 \\
\hline 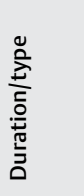 & 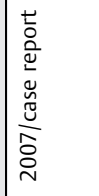 & 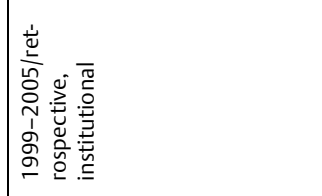 & 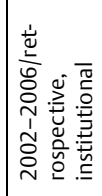 & 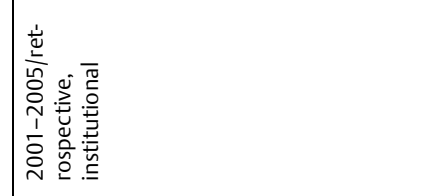 & 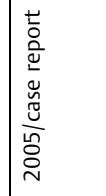 & 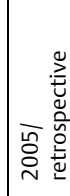 & 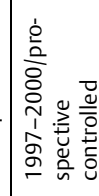 & 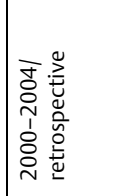 \\
\hline 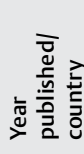 & 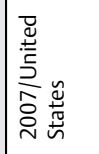 & 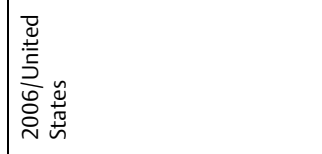 & 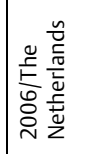 & 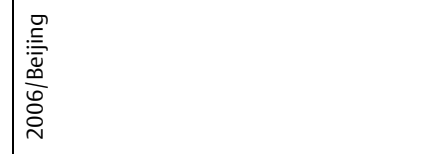 & 离 & 吂 & 离 & 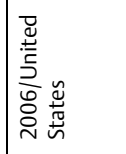 \\
\hline $\begin{array}{l}\frac{b}{2} \\
\frac{1}{2}\end{array}$ & 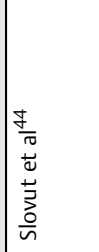 & 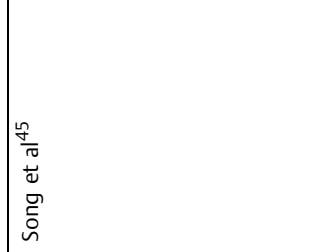 & 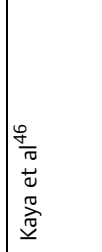 & $\begin{array}{l}\frac{\partial}{\frac{7}{00}} \\
\tilde{u} \\
\vec{x}\end{array}$ & 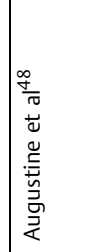 & 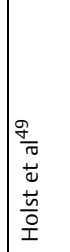 & $\begin{array}{l}\frac{0}{0} \\
\frac{n}{\pi} \\
0 \\
0 \\
\frac{\pi}{0} \\
0 \\
0\end{array}$ & 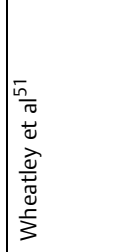 \\
\hline
\end{tabular}




\begin{tabular}{|c|c|c|c|c|c|c|c|c|c|c|}
\hline 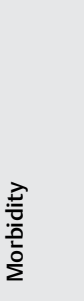 & 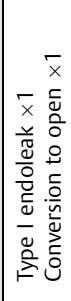 & 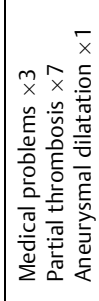 & 0 & 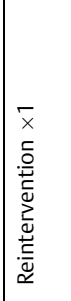 & 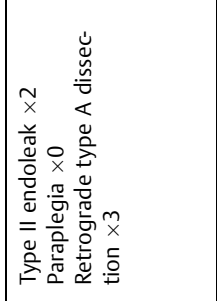 & 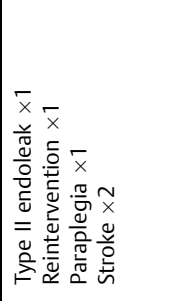 & 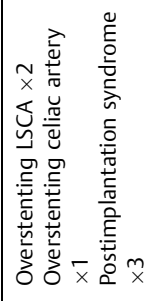 & 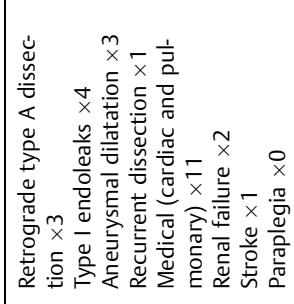 & 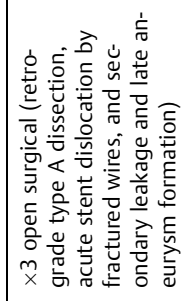 & 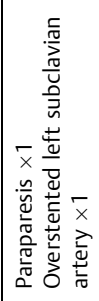 \\
\hline 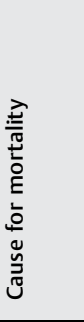 & 1 & $\tilde{z}$ & 1 & 1 & 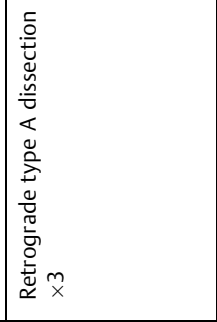 & 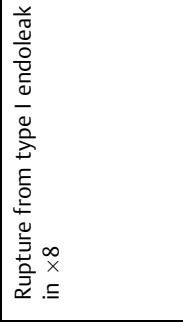 & 1 & 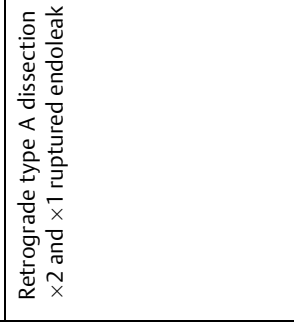 & 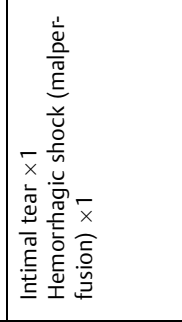 & \\
\hline 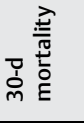 & 0 & $m$ & 0 & 0 & $m$ & $=$ & 0 & $m$ & $\sim$ & 0 \\
\hline$z$ & $\stackrel{0}{\circ}$ & $\stackrel{\infty}{\sim}$ & - & - & $\dddot{q}$ & 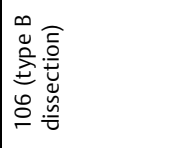 & $\infty$ & $\stackrel{\Xi}{\sim}$ & 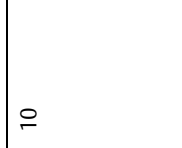 & $=$ \\
\hline 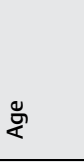 & $\tilde{z}$ & 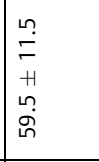 & $\bullet$ & ஸे & $\tilde{z}$ & $\tilde{z}$ & $\tilde{z}$ & $\mid \begin{array}{l}0 \\
\infty \\
0 \\
\tilde{y} \\
\delta \\
\delta\end{array}$ & 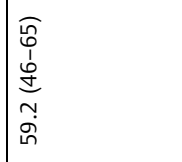 & $\tilde{z}$ \\
\hline ¿ัّ & 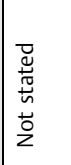 & 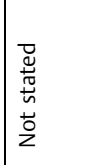 & 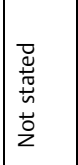 & 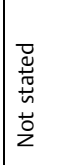 & 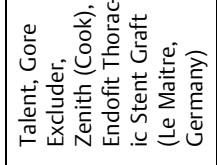 & 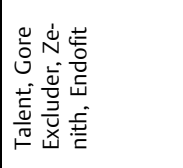 & $\Sigma$ & 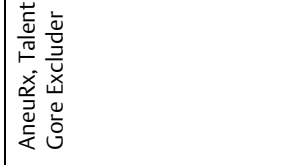 & $\frac{\overrightarrow{\widetilde{\omega}}}{\frac{\vec{\sigma}}{\Gamma}}$ & 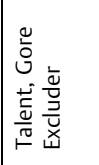 \\
\hline 鵕 & 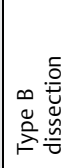 & 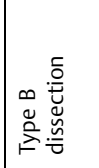 & 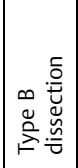 & 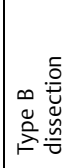 & 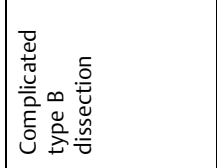 & 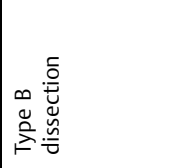 & 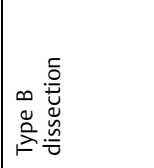 & 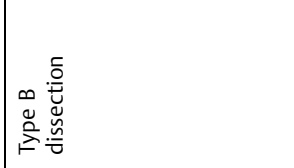 & 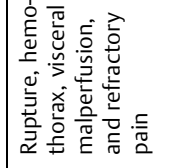 & 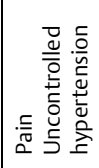 \\
\hline 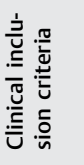 & 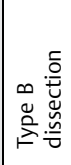 & 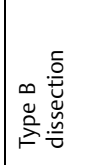 & 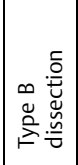 & 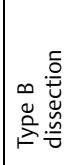 & 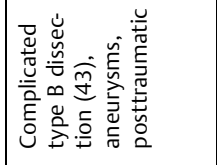 & 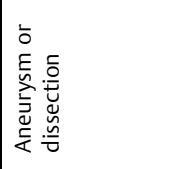 & 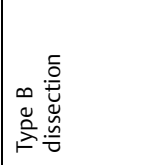 & 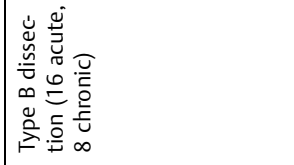 & 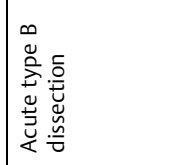 & 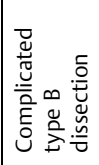 \\
\hline 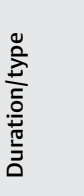 & 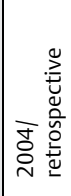 & 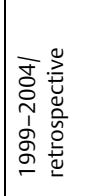 & 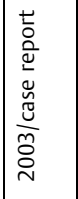 & 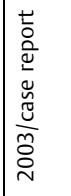 & 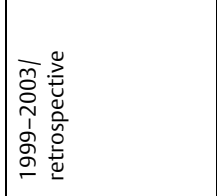 & 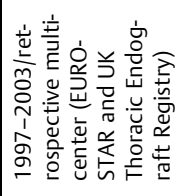 & 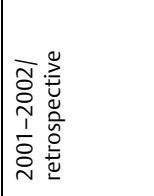 & 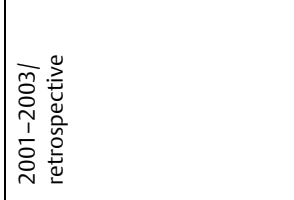 & 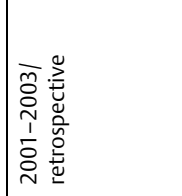 & 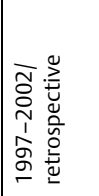 \\
\hline 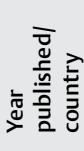 & 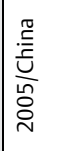 & 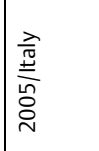 & 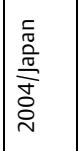 & 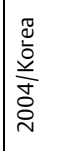 & 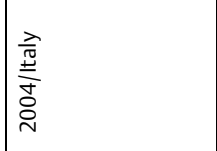 & 产 & 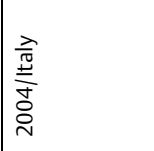 & 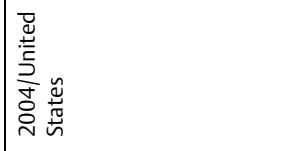 & 㤩 & 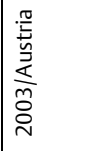 \\
\hline $\begin{array}{l}\text { 高 } \\
\text { 妾 }\end{array}$ & $\mid$ & 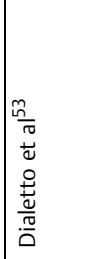 & 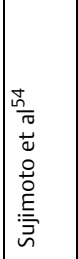 & 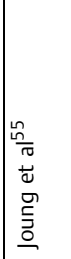 & 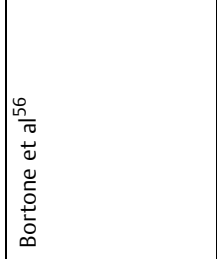 & $\stackrel{\underline{\underline{u}}}{\underline{\underline{u}}}$ & 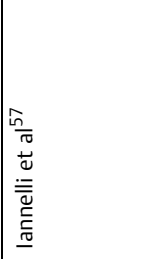 & 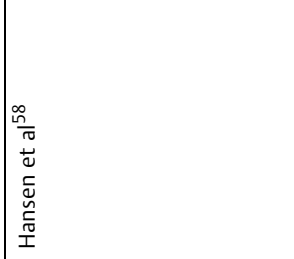 & 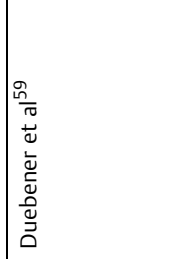 & 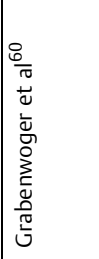 \\
\hline
\end{tabular}




\begin{tabular}{|c|c|c|c|c|c|c|c|c|c|c|c|c|}
\hline 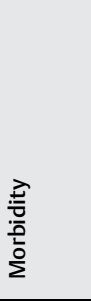 & 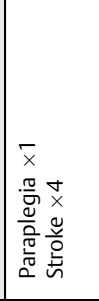 & 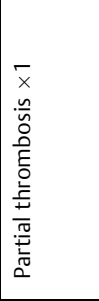 & 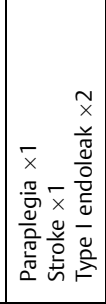 & 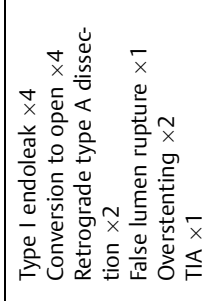 & 0 & 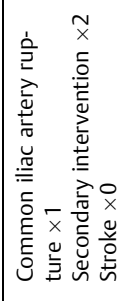 & 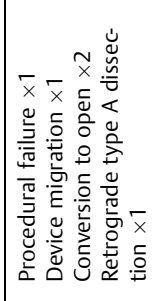 & 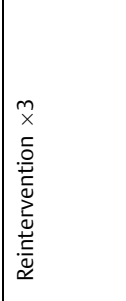 & 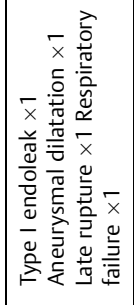 & 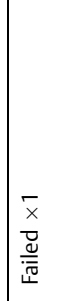 & 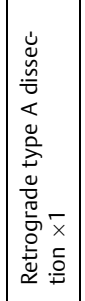 & 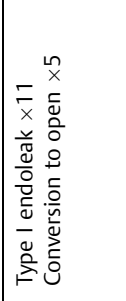 \\
\hline 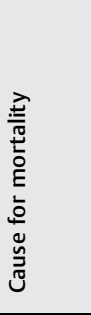 & 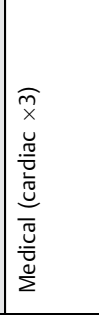 & 1 & 1 & 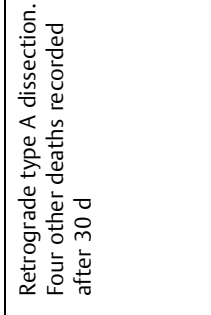 & 1 & 1 & 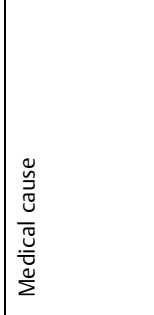 & $\begin{array}{l}\overline{0} \\
\frac{\widehat{D}}{\mathrm{~T}} \\
\bar{\Sigma} \\
\bar{\Sigma}\end{array}$ & 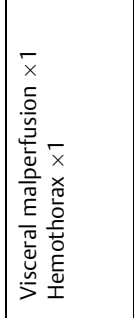 & 1 & I & 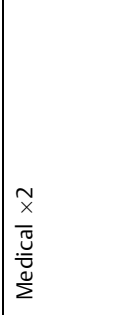 \\
\hline 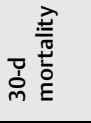 & $m$ & 0 & 0 & - & 0 & - & - & - & $\sim$ & - & 0 & $\sim$ \\
\hline$z$ & $\stackrel{\infty}{\sim}$ & $=$ & in & 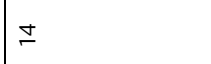 & - & $a$ & $\stackrel{n}{\longrightarrow}$ & $\stackrel{\infty}{\sim}$ & $\stackrel{\infty}{\sim}$ & $\hat{m}$ & $\stackrel{\sim}{\sim}$ & $\stackrel{\infty}{n}$ \\
\hline 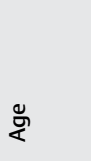 & $\tilde{z}$ & $\tilde{z}$ & $\tilde{z}$ & $\tilde{z}$ & R & $\tilde{z}$ & 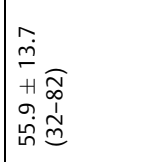 & 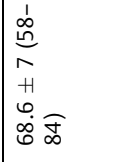 & $\tilde{z}$ & $\Sigma$ & $\tilde{z}$ & $\tilde{z}$ \\
\hline ¿ัّ & $\tilde{z}$ & 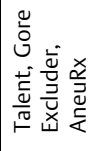 & $\tilde{z}$ & 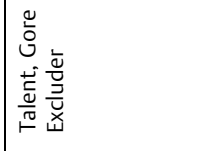 & $\tilde{z}$ & $\tilde{z}$ & $\Sigma$ & 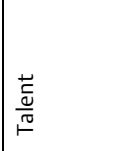 & $\Sigma$ & $\Sigma$ & $\Sigma$ & $\tilde{z}$ \\
\hline 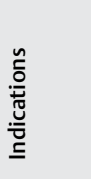 & 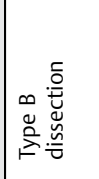 & 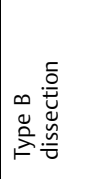 & 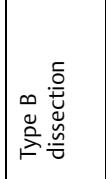 & 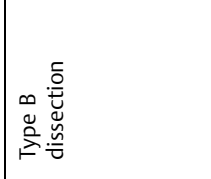 & 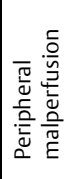 & 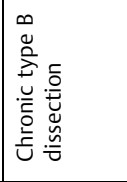 & 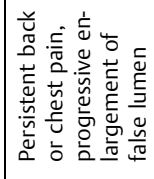 & 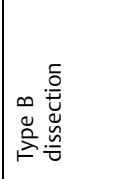 & 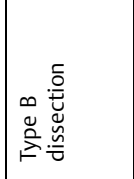 & 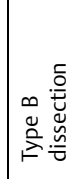 & 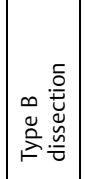 & 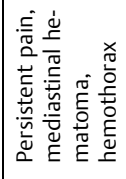 \\
\hline 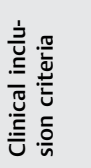 & 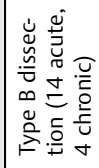 & 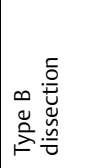 & 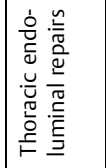 & 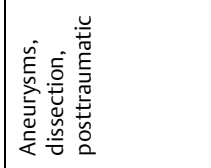 & 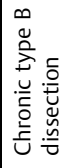 & 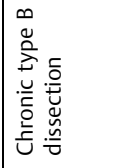 & 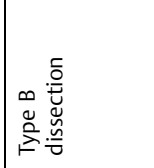 & 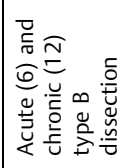 & 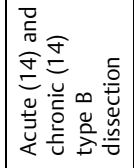 & 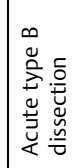 & 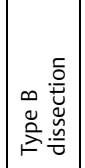 & 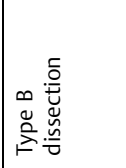 \\
\hline 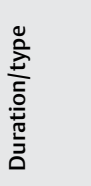 & 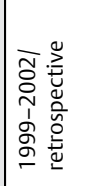 & 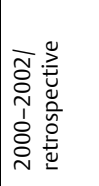 & 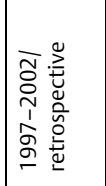 & 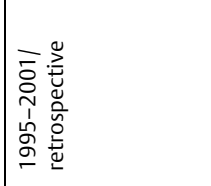 & 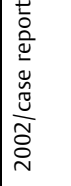 & 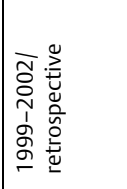 & 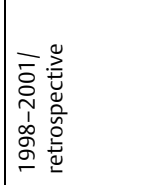 & 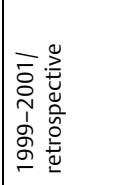 & 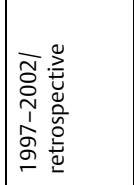 & 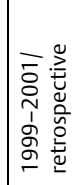 & 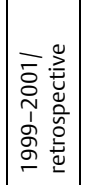 & 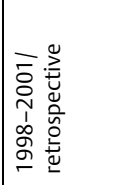 \\
\hline 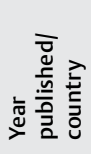 & 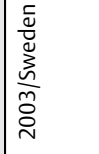 & 竞 & 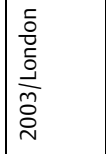 & 窟 & 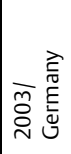 & 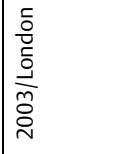 & 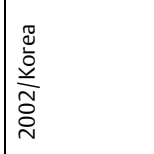 & 蒿 & 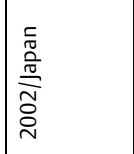 & 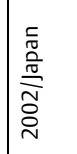 & 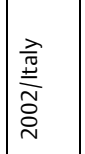 & 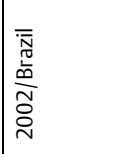 \\
\hline $\begin{array}{l}\text { 产 } \\
\text { 章 }\end{array}$ & 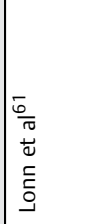 & 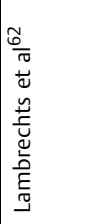 & 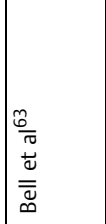 & 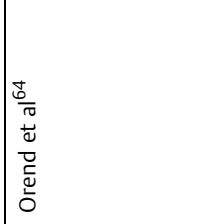 & 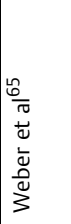 & 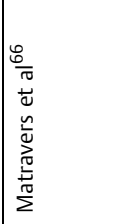 & 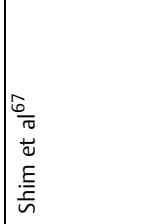 & $\mid \begin{array}{l}\frac{\infty}{0} \\
\frac{0}{\pi} \\
\frac{0}{0} \\
\frac{0}{0} \\
\frac{0}{\underline{0}} \\
\frac{1}{1}\end{array}$ & 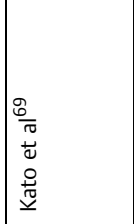 & 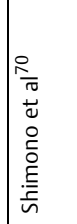 & 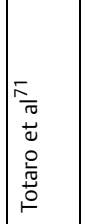 & 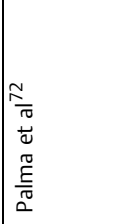 \\
\hline
\end{tabular}




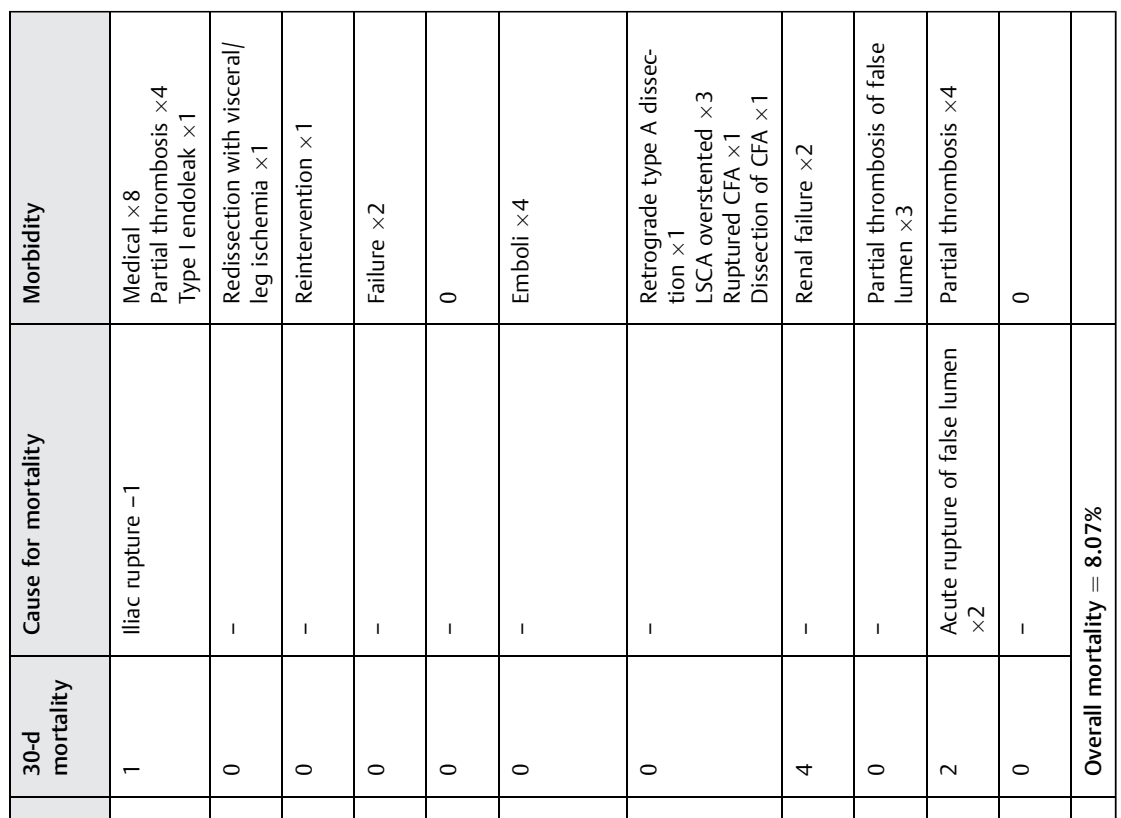

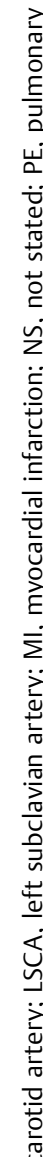

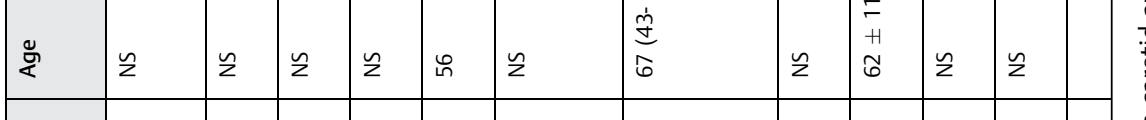

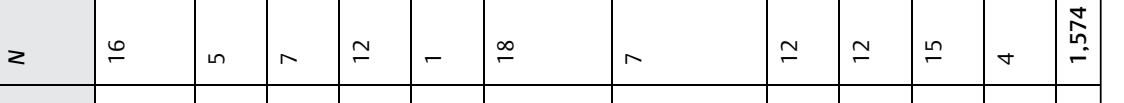

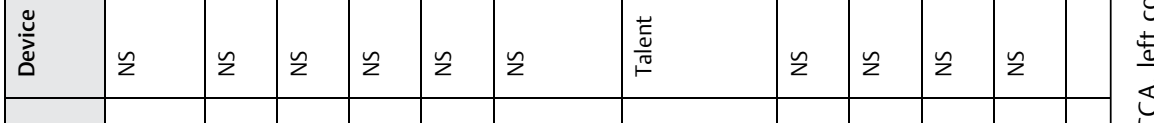

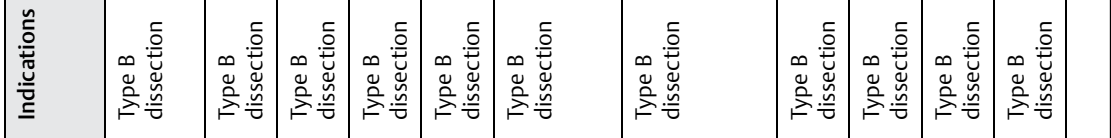

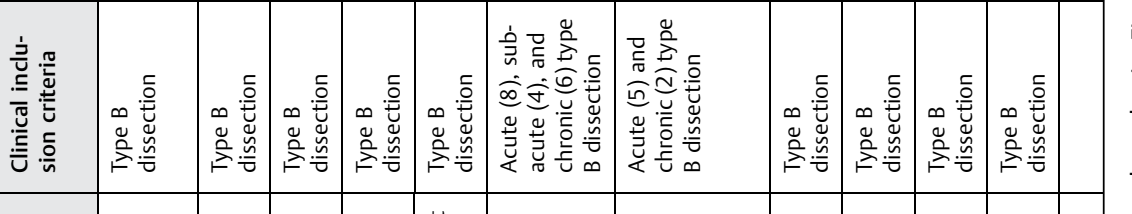

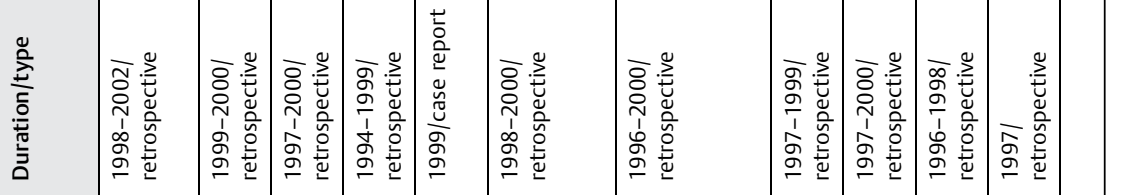


Table 2 Mortality associated with TEVAR in $<30$ days $^{\text {a }}$ (see - Table 1)

\begin{tabular}{|l|l|l|l|}
\hline Complication & $\begin{array}{l}\text { 44 studies (1998-2007) } \\
(\boldsymbol{n}=\mathbf{7 8 7} \text { patients) }\end{array}$ & $\begin{array}{l}\mathbf{2 5} \text { studies (2007-2013) } \\
(\boldsymbol{n}=\mathbf{7 8 7} \text { patients) }\end{array}$ & $\begin{array}{l}\text { Total (\%) } \\
(\boldsymbol{n}=\mathbf{1 2 1 / 1 2 7 )}\end{array}$ \\
\hline Retrograde type A dissection & 8 & 9 & $17(14 \%)$ \\
\hline $\begin{array}{l}\text { Persistent visceral and lower } \\
\text { limb malperfusion }\end{array}$ & 3 & 17 & $20(16.5 \%)$ \\
\hline $\begin{array}{l}\text { Rupture of false lumen or type I } \\
\text { endoleak or aortoesophageal } \\
\text { fistula }\end{array}$ & 17 & 30 & $47(38.8 \%)$ \\
\hline Aortoesophageal fistula & 0 & 2 & $2(1.7 \%)$ \\
\hline Further type B dissection & 0 & 2 & 2 \\
\hline Ruptured lliac or access vessel & 1 & 1 & 2 \\
\hline Open conversion & 0 & 2 & 2 \\
\hline $\begin{array}{l}\text { Device-related multiorgan } \\
\text { failure }\end{array}$ & 0 & 5 & $5(4.1 \%)$ \\
\hline $\begin{array}{l}\text { Total stent-related mortality } \\
\text { (medical causes excluded) } \\
(n=121)\end{array}$ & $29 / 121(24.0 \%)$ & $68 / 121(56.2 \%)$ & $\begin{array}{l}121(95 \%) \text { clearly } \\
\text { documented out of } \\
127 \text { mortalities }\end{array}$ \\
\hline $\begin{array}{l}\text { Medical cause (cardiac or pul- } \\
\text { monary, renal failure or stroke) }\end{array}$ & 8 & 16 & $24(12 \%)$ \\
\hline
\end{tabular}

Abbreviation: TEVAR, thoracic endovascular aortic repair.

a Rates calculated from available information of 121 out of 127 deaths.

( $p<0.05$ using Pearson chi-square test with a linear-bylinear association of $p=0.020$ ). This was contributed mainly by three patient groups: (1) the persistent visceral malperfusion/lower limb malperfusion group, (2) the ruptured false lumen/type I endoleak/aorto-esophageal fistula group, and (3) the all-cause medical-related group (-Table 2 ).

The morbidity rates revealed a similar pattern showing 36.6 versus $25.0 \%$ in the 2007 to 2013 and 1998 to 2007 groups, respectively, but did not reach statistical significance $(p>0.05)$. The main contributors to this included retrograde type A dissections, visceral malperfusion, cerebrovascular accidents, partial thrombosis of the false lumen, secondary interventions, and renal failure (-Table 3 ).

There was no major statistical difference with regard to age or stent-graft used.

\section{Discussion}

\section{Background}

Acute aortic syndrome describes the acute presentation of patients with characteristic aortic pain caused by potentially life-threatening thoracic aortic pathology. These include aortic dissection, intramural hematoma, penetrating aortic ulcer, aneurysmal leakage or rupture, and traumatic transaction. ${ }^{12}$ Aortic dissection can lead to aneurysm formation and its associated complications. The etiology is multifactorial with causes related to connective tissue or inherited disorders, developmental disorders associated with pregnancy, syphilis, crack cocaine usage, and cardiac catheterization.

Traditional management consisted of hypotensive medical management with open surgical intervention reserved for complicated cases with intractable pain, uncontrolled hyper- tension, progression of dissection, rupture, dilatation, lower limb ischemia, or visceral hypoperfusion. ${ }^{13}$ Stent-graft technology has caused a paradigm shift in the approach for symptomatic cases. This is not without major risk which includes stroke, transient ischemic attacks, paraplegia, paraparesis, distal embolization, migration, endoleak, progressive dilatation, and inadvertent covering of arch or visceral vessels. $^{14}$

\section{Literature Review}

De Bakey et $\mathrm{al}^{13}$ described their experience Between 1953 and 1993 with a total of 659 patients undergoing descending thoracic aneurysm resection. The most common cause was atherosclerosis and pain was the main presenting symptom. Perioperative mortality fell from $24.2 \%$ between 1953 and 1964 to $14.3 \%$ between 1970 and 1993 using conventional open techniques. Paraplegia occurred in $4.1 \%$, and paraparesis occurred in 5.9\%. The major source of perioperative morbidity and mortality was cardiac causes (48\%) followed by perioperative hemorrhage (14.4\%), pulmonary complications (14.4\%), and rupture of another aneurysmal segment (12.0\%). ${ }^{13}$

TEVAR has now become the standard of care for symptomatic disease in the past 10 years. The largest articles included that of Eggebrecht et al from Germany in 2005 in the European Heart Journal ${ }^{3}$ with an analysis of 609 cases collected from 39 studies from 1999 to 2004. There has also been another article published by Walsh et $\mathrm{al}^{4}$ from Cambridge, United Kingdom, in the Journal of Vascular Surgery, 2008, with 17 eligible series totaling 1,109 patients between 1991 and 1997. This particular article did not specifically address Stanford B dissection and included data with respect to aneurysm formation and traumatic rupture. 
Table 3 Morbidity associated with TEVAR

\begin{tabular}{|c|c|c|c|}
\hline Complication & $\begin{array}{l}44 \text { studies (1998-2007) } \\
\text { ( } n=787 \text { patients) }\end{array}$ & $\begin{array}{l}25 \text { studies (2007-2013) } \\
\text { ( } n=787 \text { patients) }\end{array}$ & $n=1,574(\%)$ \\
\hline $\begin{array}{l}\text { Retrograde type A } \\
\text { dissection }\end{array}$ & 21 & 28 & $49(3.1 \%)$ \\
\hline Visceral malperfusion & 5 (including 1 lower limb) & $\begin{array}{l}27 \text { (4 lower limbs with } 1 \\
\text { amputation) }\end{array}$ & $32(2.0)$ \\
\hline Aortoesophageal fistula & 0 & 4 (1 esophageal necrosis) & $4(<0.1)$ \\
\hline Cerebrovascular accident & 11 (including 1 TIA) & 30 & $41(2.7)$ \\
\hline Type I endoleak & 39 & 43 & $82(5.2)$ \\
\hline Type II endoleak & 6 & 5 & $11(<0.1)$ \\
\hline Paraplegia/paraparesis & 6 & 24 (3 hemiplegia) & $30(1.9)$ \\
\hline $\begin{array}{l}\text { Partial thrombosis of } \\
\text { false lumen }\end{array}$ & 16 & 23 & $39(2.5)$ \\
\hline Conversion to open & 16 & 14 & $30(1.9)$ \\
\hline Secondary interventions & 25 & 40 & $65(4.1)$ \\
\hline $\begin{array}{l}\text { Device migration/modu- } \\
\text { lar disconnection/junc- } \\
\text { tional leaks }\end{array}$ & 5 & 2 & $7(<0.1)$ \\
\hline Emboli & 4 & 0 & $4(<0.1)$ \\
\hline $\begin{array}{l}\text { Medical complications } \\
\text { (cardiac, pulmonary, } \\
\text { multiorgan failure) }\end{array}$ & 29 & 25 & $54(3.4)$ \\
\hline Renal failure & 8 & 23 & $31(2.0)$ \\
\hline $\begin{array}{l}\text { Overstenting celiac } \\
\text { artery }\end{array}$ & 1 & 0 & $1(<0.1)$ \\
\hline $\begin{array}{l}\text { Postimplantation } \\
\text { syndrome }\end{array}$ & 3 & 0 & $3(<0.1)$ \\
\hline Failure & 2 & 0 & $2(<0.1)$ \\
\hline Total complication rate & 197 (25.0\%) & $288(36.6 \%)$ & 485 (30.8\%) \\
\hline
\end{tabular}

Abbreviation: TEVAR, thoracic endovascular aortic repair.

Recently, the first randomized control trial was published by Nienaber et $\mathrm{al}^{7}$ in December 2009 and most of the studies have been retrospective observational studies. Nienaber et al randomized 72 patients to elective stent-graft repair with medical management and 68 patients to medical therapy alone with surveillance with the primary end point being allcause death at 2 years in uncomplicated type B aortic dissection. TEVAR failed to improve the 2 -year survival and adverse event rates despite favorable aortic remodeling., ${ }^{7,8}$

The NICE (National Institute for Clinical Excellence) guideline was based on a systematic review of published evidence commissioned by the institute with a total of 29 studies (27 case series and 2 comparative observational studies). In one comparative study, the technical success was $100 \%$ (67 patients) and overall technical success rate was $93 \%$ across 18 studies (16 case series and 2 comparative studies). The conversion to open rate varied from $0 \%$ (26 cases) to $7 \%$ ( 1 of 14 cases) and the number who developed aneurysmal dilatation varied from $0 \%$ ( 18 cases) to $7 \%$ ( 2 of 29 cases). The largest study of 84 patients showed an aneurysmal dilatation rate of $5 \%$ ( 4 of 84$)$. The number of patients who experienced a decrease in aneurysm size varied from $100 \%$ in one study to $17 \%$ in another. Of relevance to this present article, the 30-day mortality by NICE varied from $0 \%$ in several studies with a combined population of 94 patients to $14 \%$ ( 2 of 14 patients). The overall mortality ranged from 3 to $24 \%$ across 17 studies with a mean follow-up of 14 months. The most common complication was endoleak with a mean incidence of $13 \%$ over 12 months (752 patients). In five studies, there were no endoleaks reported in 83 patients. In terms of major complications, injuries to access artery occurred in nine case series and included iliac artery dissection in one, perforation of the iliac artery in one, and rupture of the femoral artery in two cases. There were six cases of stent-graft migration in 15 case series. Other complications included wound problems in $25 \%$, stroke in $19 \%$, renal failure requiring dialysis in $11 \%$, and paraplegia in $7 \%{ }^{14}$

The article of Eggebrecht et al showed procedural success in $98 \%$ and major complications in $11 \%$ including a stroke rate of $0.8 \%$ and paraplegia of $1.9 \%$. Overall complications were higher in acute dissections compared with chronic. Overall survival rates were $90 \%$ at 6 months and $88 \%$ at 2 years. ${ }^{3}$ 
A recent study by Thrumurthy et $\mathrm{a}^{15}$ in 2010 (at St. George's Vascular Institute, London) showed the role of TEVAR in chronic type B aortic dissection with reference to its midterm success. Analysis on 17 studies of 567 patients was conducted showing technical success in $89.9 \%$ and a midterm mortality of $9.2 \%$ (46/499) with a median followup of 24 months. Morbidity included an 8.1\% endoleak rate (type I) and reintervention rates ranging from 0 to $60 \%$ with a median follow-up of 31 months. Of 332 patients, 26 (7.8\%) developed aneurysms of the distal aorta or continued false lumen perfusion with aneurysmal dilatation. Rare complications included delayed retrograde type A dissection (0.67\%), aortooesophageal fistula $(0.22 \%)$, and neurological complications (paraplegia 2/447, 0.45\%; stroke $7 / 475,1.5 \%$ ). It was concluded that the absolute benefit of TEVAR over alternative treatments for chronic type B aortic dissection remains uncertain with a lack of natural history data for medically treated cases; significant heterogeneity in case selection and the absence of consensus reporting standards for intervention are significant obstructions. ${ }^{15}$

In 2010, Nienaber et al critiqued the INSTEAD trial and concluded that there was no difference in all-cause mortality at 2 years, with cumulative survival of $95 \%$ with optimal medical therapy versus $88 \%$ with TEVAR $(p=0.15)$. Aortarelated mortality was also not different $(p=0.44)$ within the first 2 years of follow-up, and risk for the combined endpoints of aorta-related death (rupture) and progression (including conversion or additional endovascular or open surgery) was similar $(p=0.65)$. They concluded that the data in the INSTEAD trial show that in the setting of clinically stable, so-called uncomplicated type B aortic dissection, elective stent-graft placement on top of optimized medical management fails to improve survival and adverse events within an observation period of 2 years, despite favorable aortic remodeling. ${ }^{16}$

Fattori et al ${ }^{17}$ in the team's consensus document examined 63 studies published from 2006 to 2012. There were 1,548 patients treated medically, 1,706 with open surgery, and 3,457 underwent TEVAR. The pooled early mortality rates (first 2 weeks) were $6.4 \%$ with medical treatment, $10.2 \%$ with TEVAR, and $17.5 \%$ with open surgery, mostly for complicated cases. Limited data for treatment of subacute (2-6 weeks after onset) type B aortic dissection showed an early mortality rate of $2.8 \%$ with TEVAR. In chronic (after 6 weeks) type B aortic dissection, 5-year survival of 60 to $80 \%$ was expected with medical therapy. If interventional treatment was applied, the pooled early mortality rate was $6.6 \%$ with TEVAR and $8.0 \%$ with open surgery. ${ }^{17}$

It seems that overall mortality and morbidity rates have been increased for TEVAR intervention for S-B-AD. This was demonstrated in the study herein presented, whereby the overall mortality and morbidity rates for TEVAR were $8.07 \%$ ( $n=127)$ and $30.8 \%(n=485)$, respectively. Additionally, the stent-graft-related mortality rate (which includes the subset of patients who died; $n=127$ ) increased in the 2007 to 2013 group (787 patients) compared with the 1998 to 2007 group (787 patients) (56.2 vs. $24 \% ; p<0.05$ ) as well as the overall morbidity rates from $25 \%$ (1998-2007) to $36.6 \%$ (2007-2013).
With respect to type I endoleak rates, this study showed a marginal increase in the leak rate from 39 in the 1998 to 2007 group to 43 in the 2007 to 2013 group with overall type I endoleak rates averaging 5.2\%. Type II endoleaks also showed few numbers with an overall leak rate of $<0.1 \%$.

Retrograde aortic dissection accounted for 3.1\% (49) of the study population with only a marginal increase from 21 to 28 in the 1998 to 2007 period and 2007 to 2013 periods, respectively. This is in keeping with most of the other studies with rates in a similar range.

Visceral malperfusion was, however, on a significant increase as seen from 5 in the 1998 to 2007 period compared with 27 cases in the 2007 to 2013 period.

This may be explained by a more aggressive clinical approach to patient selection for S-B-AD and quite possibly better reporting leading to bias from previous inadequate data collection in older studies. This has occurred in spite of overall improvement in stent-graft technology and techniques. In conclusion, the data available are still too heterogeneous to make solid conclusions and the only way forward is through better data registries and well-designed clinical trials.

\section{Conflict of Interest \\ No conflicts of interest declared.}

\section{Declaration}

This article is based on a thesis for the Masters of Science in Surgical Practice done in 2009 at the University of Kent at Canterbury, England.

\section{References}

1 Dotter CT, Judkins MP. Transluminal treatment of atherosclerotic obstruction: description of a new technique and preliminary report of this application. Circulation 1964;30:654-670

2 Parodi JC, Palmaz JC, Barone HD. Transfemoral intraluminal graft implantation for abdominal aortic aneurysms. Ann Vasc Surg 1991;5(6):491-499

3 Eggebrecht $H$, Nienaber CA, Neuhäuser $M$, et al. Endovascular stent-graft placement in aortic dissection: a meta-analysis. Eur Heart J 2006;27(4):489-498

4 Walsh SR, Tang TY, Sadat U, et al. Endovascular stenting versus open surgery for thoracic aortic disease: systematic review and meta-analysis of perioperative results. J Vasc Surg 2008;47(5): 1094-1098

5 Xiong J, Jiang B, Guo W, Wang SM, Tong XY. Endovascular stent graft placement in patients with type B aortic dissection: a metaanalysis in China. J Thorac Cardiovasc Surg 2009;138(4):865-872

6 Zhang H, Wang ZW, Zhou Z, Hu XP, Wu HB, Guo Y. Endovascular stent-graft placement or open surgery for the treatment of acute type B aortic dissection: a meta-analysis. Ann Vasc Surg 2012; 26(4):454-461

7 Nienaber CA, Rousseau H, Eggebrecht $\mathrm{H}$, et al; INSTEAD Trial. Randomized comparison of strategies for type B aortic dissection: the INvestigation of STEnt Grafts in Aortic Dissection (INSTEAD) trial. Circulation 2009;120(25):2519-2528

8 Nienaber CA, Kische S, Akin I, et al. Strategies for subacute/chronic type B aortic dissection: the Investigation Of Stent Grafts in Patients with type B Aortic Dissection (INSTEAD) trial 1-year outcome. J Thorac Cardiovasc Surg 2010;140(6):S101-S108, discussion S142-S146 
9 Fattori R, Montgomery D, Lovato L, et al. Survival after endovascular therapy in patients with type B aortic dissection: a report from the International Registry of Acute Aortic Dissection (IRAD). JACC Cardiovasc Interv 2013;6(8):876-882

10 Kische S, Ehrlich MP, Nienaber CA, et al. Endovascular treatment of acute and chronic aortic dissection: midterm results from the Talent Thoracic Retrospective Registry. J Thorac Cardiovasc Surg 2009;138(1):115-124

11 Leurs LJ, Bell R, Degrieck Y, Thomas S, Hobo R, Lundbom J; EUROSTAR; UK Thoracic Endograft Registry collaboratorsEndovascular treatment of thoracic aortic diseases: combined experience from the EUROSTAR and United Kingdom Thoracic Endograft registries. J Vasc Surg 2004;40(4):670-679, discussion 679-680

12 Ahmad F, Cheshire N, Hamady M. Acute aortic syndrome: pathology and therapeutic strategies. Postgrad Med J 2006;82(967): 305-312

13 De Bakey ME, Cooley DA, Creech O Jr. Surgical considerations of dissecting aneurysm of the aorta. Ann Surg 1955;142(4):586-610, discussion, 611-612

14 National Institute of Clinical Excellence. Endovascular stent-graft placement in thoracic aortic aneurysms and dissections. Procedure Guidance number 127; 2005

15 Thrumurthy SG, Karthikesalingam A, Patterson BO, et al. A systematic review of mid-term outcomes of thoracic endovascular repair (TEVAR) of chronic type B aortic dissection. Eur J Vasc Endovasc Surg 2011;42(5):632-647Erratum in: Eur J Vasc Endovasc Surg 2012;43(5):619

16 Nienaber CA. Influence and critique of the INSTEAD Trial (TEVAR versus medical treatment for uncomplicated type B aortic dissection). Semin Vasc Surg 2011;24(3):167-171

17 Fattori R, Cao P, De Rango P, et al. Interdisciplinary expert consensus document on management of type B aortic dissection. J Am Coll Cardiol 2013;61(16):1661-1678

18 Hanna JM, Andersen ND, Ganapathi AM, McCann RL, Hughes GC. Five-year results for endovascular repair of acute complicated type B aortic dissection. J Vasc Surg 2014;59(1):96-106

19 Wilkinson DA, Patel HJ, Williams DM, Dasika NL, Deeb GM. Early open and endovascular thoracic aortic repair for complicated type B aortic dissection. Ann Thorac Surg 2013;96(1):23-30, discussion 230

20 Minami T, Imoto K, Uchida K, et al. Clinical outcomes of emergency surgery for acute type B aortic dissection with rupture. Eur J Cardiothorac Surg 2013;44(2):360-364, discussion 364-365

21 Lombardi JV, Cambria RP, Nienaber CA, et al; STABLE investigators. Prospective multicenter clinical trial (STABLE) on the endovascular treatment of complicated type B aortic dissection using a composite device design. J Vasc Surg 2012;55(3):629-640, e2

22 Heijmen RH, Thompson MM, Fattori R, Goktay Y, Teebken OE, Orend $\mathrm{KH}$. Valiant thoracic stent-graft deployed with the new captivia delivery system: procedural and 30-day results of the Valiant Captivia registry. J Endovasc Ther 2012;19(2):213-225

23 Mitchell ME, Rushton FW Jr, Boland AB, Byrd TC, Baldwin ZK. Emergency procedures on the descending thoracic aorta in the endovascular era. J Vasc Surg 2011;54(5):1298-1302, discussion 1302

24 Fioranelli A, Razuk Filho A, Castelli Júnior V, Karakhanian W, Godoy JM, Caffaro RA. Mortality within the endovascular treatment in Stanford type B aortic dissections. Rev Bras Cir Cardiovasc 2011;26(2):250-257

25 Cambria RP, Crawford RS, Cho JS, et al; GORE TAG Investigators. A multicenter clinical trial of endovascular stent graft repair of acute catastrophes of the descending thoracic aorta. J Vasc Surg 2009; 50(6):1255-1264, e1-e4

26 Patel HJ, Williams DM, Meerkov M, Dasika NL, Upchurch GR Jr, Deeb GM. Long-term results of percutaneous management of malperfusion in acute type B aortic dissection: implications for thoracic aortic endovascular repair. J Thorac Cardiovasc Surg 2009;
138(2):300-308 [Erratum in J Thorac Cardiovasc Surg 2009;138 (3):794]

27 Day CP, Buckenham TM. Endovascular repair of the thoracic aorta: predictors of 30-day mortality in patients on the New Zealand Thoracic Aortic Stent Database (NZ TAS). Eur J Vasc Endovasc Surg 2009;37(2):160-165

28 Porcu P, Chavanon O, Thony F, Blin D. Unusual aortic stent complication after endovascular repair of the descending thoracic aorta in type B dissection in a patient with giant-cell arteritis. Interact Cardiovasc Thorac Surg 2009;8(2):287-289

29 Chemelli-Steingruber I, Chemelli A, Strasak A, et al. Endovascular repair or medical treatment of acute type B aortic dissection? A comparison. Eur J Radiol 2010;73(1):175-180

30 Mastroroberto P, di Virgilio A, Renzulli A, Indolfi C. Endovascular repair of type $B$ aortic dissection: is it possible to prevent postprocedure complications? Eur J Cardiothorac Surg 2008;33(5): 945-946, author reply 946-947

31 Flecher E, Cluzel P, Bonnet N, et al. Endovascular treatment of descending aortic dissection (type B): short- and medium-term results. Arch Cardiovasc Dis 2008;101(2):94-99

32 Apple J, McQuade KL, Hamman BL, Hebeler RF, Shutze WP, Gable DR. Initial experience in the treatment of thoracic aortic aneurismal disease with a thoracic aortic endograft at Baylor University Medical Center. Proc ( Bayl Univ Med Cent) 2008;21(2):115-119

33 Khoynezhad A, Donayre CE, Smith J, Kopchok GE, Walot I, White RA. Risk factors for early and late mortality after thoracic endovascular aortic repair. J Thorac Cardiovasc Surg 2008;135(5): 1103-1109, e1-e4

34 Dagenais F, Dumont E, Voisine P. Intramural hematoma of the arch and ascending aorta owing to acute aortic syndrome of the descending aorta: to stent or not to stent? J Endovasc Ther 2008;15(5):544-549

35 Boufi M, Hartung O, Dona B, et al. Is endovascular treatment of acute thoracic aortic disease possible in centers where extracorporal circulation is not available? [in French]. J Mal Vasc 2008; 33(2):72-78

36 Misfeld M, Sievers HH, Hadlak M, Gorski A, Hanke T. Rate of paraplegia and mortality in elective descending and thoracoabdominal aortic repair in the modern surgical era. Thorac Cardiovasc Surg 2008;56(6):342-347

37 Hager E, Moudgill N, Lipman A, Dimuzio P, Lombardi J. Coilassisted false lumen thrombosis in complicated chronic type B dissection. J Vasc Surg 2008;48(2):465-468

38 Neuhauser B, Greiner A, Jaschke W, Chemelli A, Fraedrich G. Serious complications following endovascular thoracic aortic stent-graft repair for type B dissection. Eur J Cardiothorac Surg 2008;33(1):58-63

39 Duebener L, Hartmann F, Kurowski V, et al. Surgical interventions after emergency endovascular stent-grafting for acute type B aortic dissections. Interact Cardiovasc Thorac Surg 2007;6(3): 288-292

40 Czerny M, Zimpfer D, Rodler S, et al. Endovascular stent-graft placement of aneurysms involving the descending aorta originating from chronic type B dissections. Ann Thorac Surg 2007;83(5): 1635-1639

41 Schoder M, Czerny M, Cejna M, et al. Endovascular repair of acute type B aortic dissection: long-term follow-up of true and false lumen diameter changes. Ann Thorac Surg 2007;83(3):1059-1066

42 Sharif MA, O'Donnell ME, Blair PH, Kennedy P. Emergency endovascular repair of acute descending thoracic aortic dissection. Vasc Health Risk Manag 2007;3(5):769-773

43 Bingol H, Iyem H, Akay HT, et al. Endovascular repair in management of thoracic aortic aneurysms. Int J Cardiovasc Imaging 2007; 23(1):53-59

44 Slovut DP, DeMaioribus CA, Konda S. Type B aortic dissection and left renal artery stenosis treated with a thoracic endoprosthesis. J Vasc Surg 2007;45(2):414 
45 Song TK, Donayre CE, Walot I, et al. Endograft exclusion of acute and chronic descending thoracic aortic dissections. J Vasc Surg 2006;43(2):247-258

46 Kaya A, Heijmen RH, Overtoom TT, Vos JA, Morshuis WJ, Schepens MA. Thoracic stent grafting for acute aortic pathology. Ann Thorac Surg 2006;82(2):560-565

47 Xu SD, Huang FJ, Yang JF, et al. Endovascular repair of acute type B aortic dissection: early and mid-term results. J Vasc Surg 2006; 43(6):1090-1095

48 Augustin N, Bauernschmitt R, Hausleiter J, Lange R. Dislocation of a stent-graft into the aortic arch during endovascular repair of a descending thoracic aortic aneurysm. Ann Thorac Surg 2006; 81(4):1502-1505

49 Holst AK, Quaade M, Andersen LI, Rohr N, Justesen P. Endovascular treatment of diseases of the descending thoracic aorta [in Danish]. Ugeskr Laeger 2006;168(8):781-785

50 Duda SH, Pusich B, Raygrotzki S, et al. Endovascular implantation of stent-grafts in the thoracic aorta -mid-term results of a prospective controlled study [in German]. Rofo 2002;174(4): 485-489

51 Wheatley GH III, Gurbuz AT, Rodriguez-Lopez JA, et al. Midterm outcome in 158 consecutive Gore TAG thoracic endoprostheses: single center experience. Ann Thorac Surg 2006;81(5):1570-1577, discussion 1577

52 Wang ZG, Li C. Single-branch endograft for treating Stanford type B aortic dissections with entry tears in proximity to the left subclavian artery. J Endovasc Ther 2005;12(5):588-593

53 Dialetto G, Covino FE, Scognamiglio G, et al. Treatment of type B aortic dissection: endoluminal repair or conventional medical therapy? Eur J Cardiothorac Surg 2005;27(5):826-830

54 Sugimoto I, Ohta T, Ishibashi $\mathrm{H}$, et al. Simultaneous open and endoluminal repair of ruptured abdominal and thoracic aortic aneurysms: report of a case. Surg Today 2004;34(11):961-964

55 Joung B, Ko YG, Park SH, et al. Expanding false lumen in the abdominal aorta 5 years after endovascular repair of a type B aortic dissection: successful exclusion of 3 distal re-entry sites. J Endovasc Ther 2004;11(5):577-581

56 Bortone AS, De Cillis E, D’Agostino D, de Luca Tupputi Schinosa L. Endovascular treatment of thoracic aortic disease: four years of experience. Circulation 2004;110(11, Suppl 1):II262-II267

57 Iannelli G, Piscione F, Di Tommaso L, Monaco M, Chiariello M, Spampinato $\mathrm{N}$. Thoracic aortic emergencies: impact of endovascular surgery. Ann Thorac Surg 2004;77(2):591-596

58 Hansen CJ, Bui H, Donayre CE, et al. Complications of endovascular repair of high-risk and emergent descending thoracic aortic aneurysms and dissections. J Vasc Surg 2004;40(2):228-234

59 Duebener LF, Lorenzen P, Richardt G, et al. Emergency endovascular stent-grafting for life-threatening acute type B aortic dissections. Ann Thorac Surg 2004;78(4):1261-1266, discussion 1266-1267

60 Grabenwoger M, Fleck T, Czerny M, et al. Endovascular stent graft placement in patients with acute thoracic aortic syndromes. Eur J Cardiothorac Surg 2003;23(5):788-793, discussion 793

61 Lonn L, Delle M, Falkenberg M, et al. Endovascular treatment of type B thoracic aortic dissections. J Card Surg 2003;18(6):539-544

62 Lambrechts D, Casselman F, Schroeyers P, De Geest R, D'Haenens P, Degrieck I. Endovascular treatment of the descending thoracic aorta. Eur J Vasc Endovasc Surg 2003;26(4):437-444

63 Bell RE, Taylor PR, Aukett M, Sabharwal T, Reidy JF. Results of urgent and emergency thoracic procedures treated by endoluminal repair. Eur J Vasc Endovasc Surg 2003;25(6):527-531

64 Orend KH, Scharrer-Pamler R, Kapfer X, Kotsis T, Görich J, SunderPlassmann L. Endovascular treatment in diseases of the descending thoracic aorta: 6-year results of a single center. J Vasc Surg 2003;37(1):91-99
65 Weber F, Schneider H, Körber T, Petzsch M, Rehders T, Nienaber CA. Percutaneous stents for the treatment of stenosis of descending aorta [in German]. Z Kardiol 2003;92(1):84-89

66 Matravers P, Morgan R, Belli A. The use of stent grafts for the treatment of aneurysms and dissections of the thoracic aorta: a single centre experience. Eur J Vasc Endovasc Surg 2003;26(6): 587-595

67 Shim WH, Koo BK, Yoon YS, et al. Treatment of thoracic aortic dissection with stent-grafts: midterm results. J Endovasc Ther 2002;9(6):817-821

68 Herold U, Piotrowski J, Baumgart D, Eggebrecht H, Erbel R, Jakob H. Endoluminal stent graft repair for acute and chronic type B aortic dissection and atherosclerotic aneurysm of the thoracic aorta: an interdisciplinary task. Eur J Cardiothorac Surg 2002;22(6): 891-897

69 Kato N, Shimono T, Hirano T, et al. Midterm results of stent-graft repair of acute and chronic aortic dissection with descending tear: the complication-specific approach. J Thorac Cardiovasc Surg 2002;124(2):306-312

70 Shimono T, Kato N, Yasuda F, et al. Transluminal stent-graft placements for the treatments of acute onset and chronic aortic dissections. Circulation 2002;106(12, Suppl 1):I241-I247

71 Totaro M, Mazzesi G, Marullo AG, Neri E, Fanelli F, Miraldi F. Endoluminal stent grafting of the descending thoracic aorta. Ital Heart J 2002;3(6):366-369

72 Palma JH, de Souza JA, Rodrigues Alves CM, Carvalho AC, Buffolo E. Self-expandable aortic stent-grafts for treatment of descending aortic dissections. Ann Thorac Surg 2002;73(4):1138-1141, discussion 1141-1142

73 Criado FJ, Clark NS, Barnatan MF. Stent graft repair in the aortic arch and descending thoracic aorta: a 4-year experience. J Vasc Surg 2002;36(6):1121-1128

74 Bortone AS, Schena S, Mannatrizio G, et al. Endovascular stentgraft treatment for diseases of the descending thoracic aorta. Eur J Cardiothorac Surg 2001;20(3):514-519

75 Sailer J, Peloschek P, Rand T, Grabenwöger M, Thurnher S, Lammer J. Endovascular treatment of aortic type B dissection and penetrating ulcer using commercially available stent-grafts. AJR Am J Roentgenol 2001;177(6):1365-1369

76 Won JY, Lee DY, Shim WH, et al. Elective endovascular treatment of descending thoracic aortic aneurysms and chronic dissections with stent-grafts. J Vasc Interv Radiol 2001;12(5):575-582

77 Yamazaki I, Imoto K, Ichikawa Y, Kondo J, Takanashi Y. Stent-graft treatment of type B aortic dissection involving the right aortic arch: case report. Jpn Circ J 2000;64(9):727-728

78 Shimono T, Kato N, Hirano T, Takeda K, Yada I. Early and mid-term results of endovascular stent grafting for aortic aneurysms [in Japanese]. Nippon Geka Gakkai Zasshi 1999;100(8):500-505

79 Czermak BV, Waldenberger P, Fraedrich G, et al. Treatment of Stanford type B aortic dissection with stent-grafts: preliminary results. Radiology 2000;217(2):544-550

80 Beregi JP, Prat A, Gaxotte V, Delomez M, McFadden EP. Endovascular treatment for dissection of the descending aorta. Lancet 2000;356(9228):482-483

81 Nienaber CA, Fattori R, Lund G, et al. Nonsurgical reconstruction of thoracic aortic dissection by stent-graft placement. N Engl J Med 1999;340(20):1539-1545

82 Dake MD, Kato N, Mitchell RS, et al. Endovascular stent-graft replacement for the treatment of acute aortic dissection. $\mathrm{N}$ Engl J Med 1999;340(20):1546-1552

83 da Fonseca JH, Buffolo E, Carvalho AC, et al. Utilization of selfexpandable prosthesis (stent) introduced through the femoral artery for treatment of dissection of the descending aorta [in Portuguese]. Arq Bras Cardiol 1998;70(6):389-2 